\title{
A Survey of Riemannian Contact Geometry
}

https://doi.org/10.1515/coma-2019-0002

Received July 20, 2018; accepted October 29, 2018

Abstract: This survey is a presentation of the five lectures on Riemannian contact geometry that the author gave at the conference "RIEMain in Contact", 18-22 June 2018 in Cagliari, Sardinia. The author was particularly pleased to be asked to give this presentation and appreciated the organizers' kindness in dedicating the conference to him.

Georges Reeb once made the comment that the mere existence of a contact form on a manifold should in some sense "tighten up" the manifold. The statement seemed quite pertinent for a conference that brought together both geometers and topologists working on contact manifolds, whether in terms of "tight" vs. "overtwisted" or whether an associated metric should have some positive curvature.

The first section will lay down the basic definitions and examples of the subject of contact metric manifolds. The second section will be a continuation of the first discussing tangent sphere bundles, contact structures on 3-dimensional Lie groups and a brief treatment of submanifolds. Section III will be devoted to the curvature of contact metric manifolds. Section IV will discuss complex contact manifolds and some older style topology. Section V treats curvature functionals and Ricci solitons. A sixth section has been added giving a discussion of the question of whether a Riemannian metric $g$ can be an associated metric for more than one contact structure; at the conference this was an addendum to the third lecture.

\section{Introduction and Examples}

Most of the basic material on contact manifolds is well known, so we start with these just to set the terminology and notation. By a contact manifold we mean a $C^{\infty}$ manifold $M^{2 n+1}$ together with a 1-form $\eta$ such that

$$
\eta \wedge(d \eta)^{n} \neq 0 .
$$

It is well known that given $\eta$ there exists a unique vector field $Z_{\eta}$, often denoted by geometers by $\xi$, such that

$$
d \eta\left(X, Z_{\eta}\right)=0 \text { and } \eta\left(Z_{\eta}\right)=1 .
$$

The vector field $Z_{\eta}$ is known as the Reeb vector field of the contact form $\eta$. Denote by $\mathcal{D}_{\eta}$ the contact subbundle defined by

$$
\left\{X \in T_{m} M: \eta(X)=0\right\} ;
$$

this is often denoted by topologists by $\xi$. Roughly speaking the meaning of the contact condition $\eta \wedge(d \eta)^{n} \neq 0$ is that the contact subbundle is as far from being integrable as possible. In fact the maximum dimension of an integral submanifold of $\mathcal{D}_{\eta}$ is only $n$. A one-dimensional integral submanifold of $\mathcal{D}_{\eta}$ is called an Legendre curve.

A diffeomorphism $f$ of $M^{2 n+1}$ (or between open subsets) is called a contact transformation if $f^{\star} \eta=F \eta$ for some non-vanishing function $F$ on the domain of $f$. If $F \equiv 1, f$ is called a strict contact transformation. There are many characterizations of contact transformations, for example:

$f$ is a contact transformation if and only if $X \in \mathcal{D}_{\eta}$ implies $f_{\star} X \in \mathcal{D}_{\eta}$.

^Corresponding Author: David E. Blair: Department of Mathematics, Michigan State University, East Lansing, MI 48824-1027, USA, E-mail: blair@math.msu.edu 
$f$ maps Legendre curves to Legendre curves.

$f$ maps $r$-dimensional integral submanifolds to $r$-dimensional integral submanifolds.

The simplest contact manifold is $\mathbb{R}^{2 n+1}\left(x^{1}, \ldots, x^{n}, y^{1}, \ldots, y^{n}, z\right)$ with the form

$$
\eta=d z-\sum_{i=1}^{n} y^{i} d x^{i}
$$

or $\eta=d t-\sum_{i=1}^{n} p^{i} d q^{i}$ thinking terms of classical mechanics. The classical theorem Darboux says that given any contact form $\eta$ one can always find local coordinates such that $\eta$ takes this form. For example, on the 3-dimensional torus, $T^{3}$, consider the contact form $\eta=\cos \theta^{3} d \theta^{1}+\sin \theta^{3} d \theta^{2}$, then for

$$
x=-\theta^{3}, \quad y=\theta^{1} \sin \theta^{3}-\theta^{2} \cos \theta^{3}, \quad z=\theta^{1} \cos \theta^{3}+\theta^{2} \sin \theta^{3}
$$

we have $\eta=d z-y d x$. In general, for a given contact form, it is difficult to solve the necessary PDEs to obtain such local coordinates.

There is also the notion of a contact structure or contact structure in the wider sense which is used by many topologists and can be defined in a number of ways. For example, one can put the emphasis on the field of $2 n$-planes $\mathcal{D}$ and to define the structure as a hyperplane field defined locally by a contact form, and in the overlap of coordinate neighborhoods $\mathcal{U} \cap \mathcal{U}^{\prime}, \eta^{\prime}=F \eta$ and hence $d \eta^{\prime}=d F \wedge \eta+F d \eta$ from which

$$
\eta^{\prime} \wedge\left(d \eta^{\prime}\right)^{n}=F^{n+1} \eta \wedge(d \eta)^{n} \neq 0 .
$$

Alternatively a contact manifold in the wider sense is a manifold with a differentiable structure modeled on the pseudogroup of contact transformations on $\mathbb{R}^{2 n+1}$.

The name contact (Berührungstransformation) seems to be due to Sophus Lie in 1890 [78] and is natural in view of the simple example of Huygens' principle published in 1690 [65].

Consider a wave front in the $x y$-plane and regard each of its points as a source. The envelope of all circles centered at these points with radius commensurate with the speed of propagation is a new wave front. It is determined by the points of the original curve and the slopes $p$ at these points. Plotting the corresponding curves in $x y p$-space, the mapping of tangent wave fronts to tangent wave fronts is a contact transformation, the contact form being $d y-p d x$ and in $x y p$-space the wave fronts become Legendre curves.

Before continuing let us briefly deal with a couple of notational matters. Regarding exterior algebra and differentiation and for the curvature tensor we adopt the standard conventions as given by Kobayashi and Nomizu in Volume 1 of their famous treatise [71]. For two 1-forms, $\alpha$ and $\beta$, the evaluation of their exterior product is given by

$$
\alpha \wedge \beta(X, Y)=\frac{1}{2}(\alpha(X) \beta(Y)-\alpha(Y) \beta(X)) .
$$

The coboundary formula for the exterior derivative of a 1-form is

$$
d \alpha(X, Y)=\frac{1}{2}(X \alpha(Y)-Y \alpha(X)-\alpha([X, Y])) .
$$

Also we will generally precede the coordinate expression of a contact form by a factor of $\frac{1}{2}$. Finally for the curvature tensor

$$
R_{X Y} Z=\nabla_{X} \nabla_{Y} Z-\nabla_{Y} \nabla_{X} Z-\nabla_{[X, Y]} Z
$$

As mentioned already the contact condition implies the maximum degree of non-integrability of the contact subbundle. One can also think of the contact condition as meaning that the corresponding hyperplane field rotates as one moves around on the manifold. So one can think of associated metrics as providing a measure of this rotation or of the corresponding Reeb vector field. An associated metric also provides contact 
geometry with a geometric structure analogous to that of an almost complex structure and Hermitian metric in symplectic geometry, i.e. an almost Kähler structure.

A Riemannian metric $g$ is an associated metric for a contact form $\eta$ if, first of all,

$$
\eta(X)=g\left(X, Z_{\eta}\right)
$$

and secondly, there exists a field of endomorphisms $\phi$ such that

$$
\phi^{2}=-I+\eta \otimes Z_{\eta} \text { and } d \eta(X, Y)=g(X, \phi Y) .
$$

In particular the contact subbundle is orthogonal to the Reeb vector field, $\phi Z_{\eta}=0, \eta \circ \phi=0$ and $\phi$ acts as an almost complex structure on $\mathcal{D}_{\eta}$. Notice the similarity to an almost Hermitian structure on a symplectic manifold. We refer to $\left(\phi, Z_{\eta}, \eta, g\right)$ as a contact metric structure and to $M^{2 n+1}$ with such a structure as a contact metric manifold.

We will also need an additional tensor field that plays a fundamental role, namely

$$
h=\frac{1}{2} \$_{Z_{\eta}} \phi
$$

where $\$$ denotes Lie differentiation. The operator $h$ is a symmetric, it anti-commutes with $\phi, h Z_{\eta}=0$ and vanishes if and only if the Reeb vector field is Killing. An important property of $h$ is the following

$$
\nabla_{X} Z_{\eta}=-\phi X-\phi h X
$$

which reflects the rotation of the Reeb vector field and in turn, by orthogonality, of the contact subbundle. An immediate consequence is that the integral curves of $Z_{\eta}$ are geodesics.

Associated metrics can be constructed in the following manner. Let $\overline{\bar{g}}$ be any Riemannian metric on $M^{2 n+1}$ and define a metric $\bar{g}$ by

$$
\bar{g}(X, Y)=\overline{\bar{g}}\left(-X+\eta(X) Z_{\eta},-Y+\eta(Y) Z_{\eta}\right)+\eta(X) \eta(Y) .
$$

Then $\bar{g}\left(X, Z_{\eta}\right)=\eta(X)$. Now choose a local $\bar{g}$-orthonormal basis $\left\{X_{1}, \ldots, X_{2 n}\right\}$ of $\mathcal{D}_{\eta}$ and evaluate $d \eta$ on these vectors. This gives a $2 n \times 2 n$ non-singular matrix, $A_{i j}=d \eta\left(X_{I}, X_{j}\right)$ which by polarization can be written as the product of an orthogonal matrix $F$ and a positive definite symmetric matrix $G$. Define an associated metric $g$ and almost complex structure $\phi$ on $\mathcal{D}_{\eta}$ by $g\left(X_{i}, X_{j}\right)=G_{i j}$ and $\phi X_{i}=F_{i}{ }^{j} X_{j}$ and extend to all tangent vectors by $g\left(X, Z_{\eta}\right)=\eta(X)$ and $\phi Z_{\eta}=0$. If now $\left\{Y_{1}, \ldots, Y_{2 n}\right\}$ is another $\bar{g}$-orthonormal basis of $\mathcal{D}_{\eta}$, There exists an orthogonal matrix $P$ such that

$$
B_{i j}=d \eta\left(Y_{i}, Y_{j}\right)=d \eta\left(P^{k}{ }_{i} X_{k}, P_{j}^{l} X_{l}\right)=\left(P A P^{-1}\right)_{i j} .
$$

If $B=\Phi \Gamma$ is the polar decomposition of $B, \Phi \Gamma=P F P^{-1} P G P^{-1}$ and so by the uniqueness of the polar decomposition $\Phi=P F P^{-1}$ and $\Gamma=P G P^{-1}$ and we have that $g$ and $\phi$ are globally defined. Now $A^{T}=G F^{T}=-F G$ and hence $G=-F F F^{T} G F$. But $F^{T} G F$ is positive definite symmetric and by the uniqueness of the polar decomposition $F^{2}=-I$ and $F=-F^{T}$. This construction is due to Hatakayama [60] in the course of which he proved the analyticity of the polar decomposition.

Since the metric $\overline{\bar{g}}$ is totally arbitrary, the procedure can become overly complicated. A variation of this idea is that if the form is presented explicitly enough, one might try to construct $2 n$ local vector fields $\left\{X_{1}, \ldots, X_{2 n}\right\}$ spanning $\mathcal{D}_{\eta}$ over a neighborhood $U$ and such that the matrix $d \eta\left(X_{i}, X_{j}\right)$ is relatively simple to polarize. Then in the above procedure define the initial metric by declaring $\left\{X_{1}, \ldots, X_{2 n}\right\}$ together with $Z_{\eta}$ to be orthonormal and use this as $\bar{g}$ giving an associated metric $g$ locally. Then $\bar{g}$ can be extended and the above procedure used to give a global associated metric which would agree with $g$ on at worst a smaller neighborhood contained in $\mathrm{U}$.

The space $\mathcal{A}$ of all associated metrics for a given contact form is infinite dimensional, so they are far from unique but they do all have the same volume element which will play a role in Section V. 
For future use we define a $\phi$-basis. Let $\mathcal{U}$ be a coordinate neighborhood on $M^{2 n+1}$ and $X_{1}$ any unit vector field on $U$ orthogonal to $Z_{\eta}$. Then $X_{1^{*}}=\phi X_{1}$ is a unit vector field orthogonal to both $X_{1}$ and $Z_{\eta}$. Now choose a unit vector field $X_{2}$ orthogonal to $Z_{\eta}, X_{1}$ and $X_{1^{*}}$. Then $\phi X_{2}$ is also a unit vector field orthogonal to $Z_{\eta}, X_{1}, X_{1^{*}}$ and $X_{2}$. Proceeding in this way we obtain a local orthonormal basis $\left\{X_{i}, X_{i^{\star}}=\phi X_{i}, Z_{\eta}\right\}, i=1, \ldots, n$, called a $\phi$-basis.

An almost contact structure consists of a field of endomorphisms $\phi$, a 1-form $\eta$ and a vector field $Z_{\eta}$ satisfying

$$
\phi^{2}=-I+\eta \otimes Z_{\eta} \text { and } \eta\left(Z_{\eta}\right)=1 .
$$

A Riemannian metric $g$ is said to be compatible if

$$
g(\phi X, \phi Y)=g(X, Y)-\eta(X) \eta(Y)
$$

and we refer to all of this as an almost contact metric structure. This is equivalent to the reduction of the structural group to $U(n) \times 1$.

We remark that it is possible to have an almost contact metric structure $\left(\phi, Z_{\eta}, \eta, g\right)$ with $\eta$ a contact form and $Z_{\eta}$ its Reeb vector field but which is not a contact metric structure. A hypersurface of an almost Hermitian manifold inherits an almost contact structure. Thus for example, the standard contact structure on $S^{5}$ can be obtained by considering it as a hypersurface of $\mathbb{C}^{n} ; S^{5}$ also inherits an almost contact structure as the equatorial hypersurface of the nearly Kähler manifold $S^{6}$ which not strictly a contact metric structure (details can be found in example (4.5.3) in [10]).

The product $M^{2 n+1} \times \mathbb{R}$ carries a natural almost complex structure defined by

$$
J\left(X, f \frac{d}{d t}\right)=\left(\phi X-f Z_{\eta}, \eta(X) \frac{d}{d t}\right)
$$

where $f$ is a function on $M^{2 n+1} \times \mathbb{R}$. The underlying almost contact structure is said to be normal if $J$ is integrable. The normality condition can be expressed as $N=0$ where $N$ is defined by

$$
N(X, Y)=[\phi, \phi](X, Y)+2 d \eta(X, Y) Z_{\eta},
$$

$[\phi, \phi]$ being the Nijenhuis tensor of $\phi$.

Definition: A Sasakian manifold is a normal contact metric manifold.

A common alternate approach to this definition is the following. Let $\left(M^{m}, g\right)$ be a Riemannian manifold, $\mathbb{R}_{+}$the positive reals and

$$
C\left(M^{m}\right)=\left(\mathbb{R}_{+} \times M^{m}, d r^{2}+r^{2} g\right)
$$

the cone over $M^{m}$. Then $\left(M^{m}, g\right)$ is Sasakian if and only if the holonomy group of $C\left(M^{m}\right)$ reduces to a subgroup of $U\left(\frac{m+1}{2}\right)$. Thus $\left(\mathbb{R}_{+} \times M^{m}, d r^{2}+r^{2} g\right)$ is Kähler and $m=2 n+1, n \geq 1$.

In terms of the covariant derivative of $\phi$ the Sasakian condition is

$$
\left(\nabla_{X} \phi\right) Y=g(X, Y) Z_{\eta}-\eta(Y) X
$$

which can be viewed as a contact metric analogue of the Kähler condition, $\nabla J=0$. In terms of the curvature tensor a contact metric structure is Sasakian if and only if

$$
R_{X Y} Z_{\eta}=\eta(Y) X-\eta(X) Y .
$$

A contact metric structure for which $Z_{\eta}$ is a Killing vector field is said to be K-contact and it is easy to see that a Sasakian manifold is K-contact. In dimension 3 the K-contact condition implies that the manifold is Sasakian but this is not true in higher dimensions.

As mentioned before, the simplest contact manifold is $\mathbb{R}^{2 n+1}\left(x^{1}, \ldots, x^{n}, y^{1}, \ldots, y^{n}, z\right)$ with the form

$$
\eta=\frac{1}{2}\left(d z-\sum_{i=1}^{n} y^{i} d x^{i}\right) .
$$


The Reeb vector field $Z_{\eta}$ is $2 \frac{\partial}{\partial z}$ and the contact subbundle $\mathcal{D}_{\eta}$ is spanned by $X_{i}=\frac{\partial}{\partial x^{i}}+y^{i} \frac{\partial}{\partial z}, X_{n+i}=\frac{\partial}{\partial y^{i}}$, $i=1, \ldots, n$. The Riemannian metric

$$
g=\eta \otimes \eta+\frac{1}{4} \sum_{i=1}^{n}\left(\left(d x^{i}\right)^{2}+\left(d y^{i}\right)^{2}\right)
$$

gives a contact metric structure on $\mathbb{R}^{2 n+1}$. The tensor field $\phi$ is given by the matrix

$$
\left(\begin{array}{ccc}
0 & \delta_{i j} & 0 \\
-\delta_{i j} & 0 & 0 \\
0 & y^{j} & 0
\end{array}\right)
$$

and the vector fields $X_{i}=2 \frac{\partial}{\partial y^{i}}, X_{n+i}=2\left(\frac{\partial}{\partial x^{i}}+y^{i} \frac{\partial}{\partial z}\right), i=1, \ldots, n$ and $Z_{\eta}$ form a $\phi$-basis for the contact metric structure.

This Riemannian metric has the following properties. The structure is Sasakian, so in particular the Reeb vector field $Z_{\eta}$ is a Killing vector field. The sectional curvature of any plane section containing $Z_{\eta}$ is equal to 1. The sectional curvature of a plane section spanned by a vector $X$ orthogonal to $Z_{\eta}$ and $\phi X$ is equal to -3; for this reason this example is often denoted $\mathbb{R}^{2 n+1}(-3)$.

In dimension 3 this example is often identified with the Heisenberg group

$$
H_{\mathbb{R}}=\left\{\left(\begin{array}{ccc}
1 & y & z \\
0 & 1 & x \\
0 & 0 & 1
\end{array}\right) \mid x, y, z \in \mathbb{R}\right\} ;
$$

left translation preserves $\eta$ and $g$ is a left invariant metric on $H_{\mathbb{R}}$.

In some sense, the beginning of the modern theory of contact manifolds is the celebrated Boothby-Wang fibration of 1958 [23]. Earlier, of course, there were many results in classical mechanics related to contact transformations and some results by Reeb [96], Chern [28] and J. Gray [54] in the 1950s were also significant. To describe the Boothby-Wang Theorem first recall that a vector field on a manifold is regular if every point has a neighborhood such that any integral curve of the vector field passing through the neighborhood, passes through only once. Two well known examples of non-regular vector fields are the irrational flow on a torus and the flow around a Möbius band.

Theorem 1. Let $\left(M^{2 n+1}, \eta^{\prime}\right)$ be a compact regular contact manifold, then there exists a contact form $\eta=\tau \eta^{\prime}$ for some non-vanishing function $\tau$ whose Reeb vector field $Z_{\eta}$ generates a free effective $S^{1}$ action on $M^{2 n+1}$. Moreover $M^{2 n+1}$ is the bundle space of a principal circle bundle $\pi: M^{2 n+1} \longrightarrow M^{2 n}$ over a symplectic manifold $M^{2 n}$ whose symplectic form $\Omega$ determines an integral cocycle on $M^{2 n}$ and $\eta$ is a connection form on the bundle with curvature form $d \eta=\pi^{\star} \Omega$.

A particular example is that of the Hopf fibration $S^{2 n+1} \longrightarrow P \mathbb{C}^{n}$ which gives a natural Sasakian structure on an odd-dimensional sphere of constant curvature +1 . More generally if the base manifold is Kähler, then the bundle space will be Sasakian.

Before leaving the topic of Sasakian manifolds let us remark that Hansjörg Geiges [47] has a nice result here which will be mentioned again in Section $\mathrm{V}$.

Theorem 2. A compact 3-dimensional manifold admits a Sasakian structure if and only if it is diffeomorphic to a left invariant quotient of $S U(2)$, the Heisenberg group or $\widetilde{S L}(2, \mathbb{R})$ by a discrete group.

We should not leave the topic of normality without discussing the relation between contact metric structures (\& almost contact structures) and CR-structures. Let $N$ be an $m(=2 n+k)$-dimensional $C^{\infty}$ manifold and $\mathcal{H}$ be a $C^{\infty}$ complex subbundle of the complexified tangent bundle of complex dimension $n$. A CR-manifold, as 
introduced by Greenfield [55], of real dimension $m$ and CR-dimension $n$ is a pair $(N, \mathcal{H})$ such that $\mathcal{H}_{p} \cap \overline{\mathcal{H}}_{p}=0$ and $\mathcal{H}$ is involutive, i.e. for vector fields $X, Y \in \mathcal{H},[X, Y] \in \mathcal{H}$. Then there exists a unique subbundle $\mathcal{D}$ of $T N$ such that $\mathcal{D}^{\mathbb{C}}=\mathcal{H} \oplus \overline{\mathcal{H}}$, and a unique bundle map $\mathcal{J}: \mathcal{D} \longrightarrow \mathcal{D}$ such that $\mathcal{J}^{2}=-I$ and $\mathcal{H}=\{X-i \mathcal{J} X \mid X \in \mathcal{D}\}$.

Now let $(M, \mathcal{H})$ be a CR-manifold with $M$ of real dimension $2 n+1$ and $\mathcal{H}$ of complex dimension $n$. Consider the space of all covectors in $T_{x}^{\star} M$ such that $\mathcal{D}$ lies in their kernel. This defines a real line bundle $F \subset T^{\star} M$. If $M$ is orientable, then $F \longrightarrow M$ admits a global nowhere vanishing section $\eta$ which is called a pseudo-Hermitian structure and $(M, \mathcal{H}, \eta)$ is called a pseudo-Hermitian manifold. The Levi form is defined by

$$
L_{\eta}(X, Y)=-d \eta(X, \mathcal{\partial} Y), \quad X, Y \in \mathcal{D} .
$$

If $L_{\eta}$ is non-degenerate, then $\eta$ is a contact form and its Reeb vector field $Z_{\eta}$ is transverse to $\mathcal{D}$. $(M, \mathcal{H}, \eta)$ is said to be strongly pseudo-convex if $L_{\eta}$ is positive definite.

Using the direct sum decomposition $T M=\mathcal{D} \oplus\left\{Z_{\eta}\right\}$ we may extend $L_{\eta}$ to a Riemannian metric $g_{\eta}$ on $M$, called the Webster metric, by $g_{\eta}\left(Z_{\eta}, Z_{\eta}\right)=1, g_{\eta}\left(Z_{\eta}, X\right)=0$ for $X \in \mathcal{D}$ and $g_{\eta}(X, Y)=L_{\eta}(X, Y)$ for $X, Y \in \mathcal{D}$. Moreover we may extend $\mathcal{J}$ to a tensor field $\phi$ on $M$ by $\phi Z_{\eta}=0$ and $\phi X=\mathcal{J} X$ for $X \in \mathcal{D}$. Therefore a strongly pseudo-convex $\mathrm{CR}$ manifold $(M, \mathcal{H}, \eta)$, carries a contact metric structure $\left(\phi, Z_{\eta}, \eta, g_{\eta}\right)$.

Turning to the case of almost contact structures, consider an almost contact manifold $M^{2 n+1}$ with structure tensors $\left(\phi, Z_{\eta}, \eta\right)$. Since $\phi^{2}=-I+\eta \otimes Z_{\eta}$ and $\phi Z_{\eta}=0$, the eigenvalues of $\phi$ are 0 and $\pm i$ each with multiplicity $n$; in particular $\phi$ is an almost complex structure on the subbundle $\mathcal{D}$ defined by $\eta=0$. Thus the complexification of $\mathcal{D}_{p}$ is decomposable as $\mathcal{D}_{p}^{\prime} \oplus \mathcal{D}_{p}^{\prime \prime}$ where $\mathcal{D}_{p}^{\prime}=\left\{X-i \phi X \mid X \in \mathcal{D}_{p}\right\}$ and $\mathcal{D}_{p}^{\prime \prime}=\left\{X+i \phi X \mid X \in \mathcal{D}_{p}\right\}$, the eigenspaces of $\pm i$ respectively.

We now present a theorem due to Ianus [66] that a normal almost contact manifold is a CR-manifold. The converse is not true and we will see below a necessary and sufficient condition for a contact metric manifold to be a CR-manifold.

Theorem 3. If $M^{2 n+1}\left(\phi, Z_{\eta}, \eta, g\right)$ is a normal almost contact manifold, then $\left(M^{2 n+1}, \mathcal{D}^{\prime}\right)$ is a CR-manifold.

For the proof, note first that $\overline{\mathcal{D}}_{p}^{\prime}=\mathcal{D}_{p}^{\prime \prime}$ and $\mathcal{D}_{p}^{\prime} \cap \mathcal{D}_{p}^{\prime \prime}=0$; thus the proof is to show that $[X-i \phi X, Y-i \phi Y] \in \mathcal{D}^{\prime}$ for $X, Y \in \mathcal{D}$. The normality condition, $[\phi, \phi]+2 d \eta \otimes Z_{\eta}=0$, then provides the necessary ingredients for the computation.

On a contact metric manifold $M^{2 n+1},\left(M^{2 n+1}, \mathcal{D}^{\prime}\right)$ might be CR without the structure being normal and Tanno [108] gave a necessary and sufficient condition for a contact metric manifold to be a CR-manifold; note the operator $h$ in this theorem.

Theorem 4. Let $\left(M^{2 n+1}, \eta, g\right)$ be a contact metric manifold. Then the pair $\left(M^{2 n+1}, \mathcal{D}^{\prime}\right)$ is a (strongly pseudoconvex) CR-manifold if and only if

$$
\left(\nabla_{X} \phi\right) Y=g(X+h X, Y) Z_{\eta}-\eta(Y)(X+h X) .
$$

Before going on we should include the idea of a $\mathcal{D}$-homothetic deformation. Given a contact metric structure $\left(\phi, Z_{\eta}, \eta, g\right)$, consider the deformed structure

$$
\bar{\eta}=a \eta, \quad \bar{Z}_{\bar{\eta}}=\frac{1}{a} Z_{\eta}, \quad \bar{\phi}=\phi, \quad \bar{g}=a g+a(a-1) \eta \otimes \eta
$$

where $a$ is a positive constant. Such a deformation is called a D-homothetic deformation, since the metrics restricted to the contact subbundle $\mathcal{D}$ are homothetic. This deformation was introduced by Tanno [106] and has many applications. Such a change preserves the states of being contact metric, K-contact, Sasakian and strongly pseudo-convex CR.

Finally we should mention the important result of Martinet [81] that a compact orientable 3-dimensional manifold always admits a contact form. Gonzalo [53] showed that there are three independent contact forms. A nice generalization of this to higher dimensions was given by Geiges and Gonzalo [49]. 
Theorem 5. If a compact contact manifold $M^{2 n+1}$ admits $k$ everywhere linearly independent vector fields belonging to the contact subbundle, then $M^{2 n+1}$ admits $(k+1)$ everywhere linearly independent contact forms with everywhere linearly independent Reeb vector fields.

In the author's view there is a significant difference between dimension 3 and dimensions $\geq 5$ in both contact geometry and contact topology; the author hopes that both dimensional settings will be treated by geometers and topologists in their future research.

\section{Tangent Sphere Bundles, Lie Groups, and Submanifolds}

\subsection{Tangent Sphere Bundles}

A second large class of contact metric manifolds is that of the tangent sphere bundles, $T_{1} M$. Despite the example of $T_{1} S^{2} \cong \mathbb{R} P^{3}$, the standard contact metric structures on tangent sphere bundles are almost never Sasakian. In fact the standard contact metric structure on the tangent sphere bundle is K-contact if and only if the base manifold has positive constant curvature +1 (Tashiro [110]). However, in general, the tangent sphere bundles are classically an important class of contact manifolds.

To describe the structure of interest here, we first review the geometry of the tangent bundle. Let $M$ be an (n+1)-dimensional $C^{\infty}$ manifold and $\bar{\pi}: T M \longrightarrow M$ its tangent bundle. If $\left(x^{1}, \ldots, x^{n+1}\right)$ are local coordinates on $M$, set $q^{i}=x^{i} \circ \bar{\pi}$; then $\left(q^{1}, \ldots, q^{n+1}\right)$ together with the fibre coordinates $\left(v^{1}, \ldots, v^{n+1}\right)$ form local coordinates on $T M$.

If $X$ is a vector field on $M$, its vertical lift $X^{V}$ on $T M$ is the vector field defined by $X^{V} \omega=\omega(X) \circ \bar{\pi}$ where $\omega$ is a 1-form on $M$, which on the left side of this equation is regarded as a function on TM. For an affine connection $D$ on $M$, the horizontal lift $X^{H}$ of $X$ is defined by $X^{H} \omega=D_{X} \omega$ and we then have the connection map $K: T T M \longrightarrow T M$ defined by

$$
K X^{H}=0, \quad K X_{t}^{V}=X_{\bar{\pi}(t)}, t \in T M .
$$

$T M$ admits an almost complex structure $J$ defined by

$$
J X^{H}=X^{V}, J X^{V}=-X^{H} .
$$

Computing the Nijenhuis torsion of $J$ one can easily see that $J$ is integrable if and only if $D$ has vanishing curvature and torsion (Hsu [62], Dombrowski [39]).

If now $G$ is a Riemannian metric on $M$ and $D$ its Levi-Civita connection, we define a Riemannian metric $g_{s}$ on TM called the Sasaki metric (not to be confused with a Sasakian structure), by

$$
g_{s}(X, Y)=\left(G\left(\bar{\pi}_{\star} X, \bar{\pi}_{\star} Y\right)+G(K X, K Y)\right) \circ \bar{\pi}
$$

where $X$ and $Y$ are vector fields on $T M$. Since $\bar{\pi}_{\star} \circ J=-K$ and $K \circ J=\bar{\pi}_{\star}, g_{s}$ is Hermitian for the almost complex structure $J$.

On TM define the Liouville form $\beta$ by $\beta(X)_{t}=G\left(t, \bar{\pi}_{\star} X\right), t \in T M$ or equivalently by the local expression $\beta=\sum G_{i j} v^{i} d q^{j}$. Then $d \beta$ is a symplectic structure on $T M$ and in particular $2 d \beta$ is the fundamental 2-form of the almost Hermitian structure $\left(J, g_{s}\right)$. Thus $T M$ has an almost Kähler structure; it is Kählerian if and only if $(M, G)$ is flat (Tachibana and Okumura [102]).

The tangent sphere bundle, $\pi: T_{1} M \longrightarrow M$, is the hypersurface of $T M$ defined by

$$
\sum G_{i j} v^{i} v^{j}=1
$$

The vector field $v=v^{i} \frac{\partial}{\partial v^{i}}$ is a unit normal as well as the position vector for a point $t \in T_{1} M$. Denote by $g^{\prime}$ the Riemannian metric induced on $T_{1} M$ from the Sasaki metric $g_{s}$ on $T M$. 
We know that as a hypersurface of the almost Kähler manifold $T M, T_{1} M$ inherits an almost contact metric structure. Following usual procedures we define $\phi^{\prime}, Z^{\prime}$ and $\eta^{\prime}$ by on $T_{1} M$ by

$$
Z^{\prime}=-J v, \quad J X=\phi^{\prime} X+\eta^{\prime}(X) v .
$$

$\left(\phi^{\prime}, Z^{\prime}, \eta^{\prime}, g^{\prime}\right)$ is then an almost contact metric structure. Moreover $\eta^{\prime}$ is the form on $T_{1} M$ induced from the Liouville form $\beta$ on $T M$, for

$$
\eta^{\prime}(X)=g_{s}(v, J X)=2 d \beta(v, X)=2 \sum d\left(G_{i j} v^{j}\right) \wedge d q^{i}\left(v^{k} \frac{\partial}{\partial v^{k}}, X\right)=\sum G_{i j} v^{j} d q^{i}(X)=\beta(X) .
$$

However $g^{\prime}\left(X, \phi^{\prime} Y\right)=2 d \eta^{\prime}(X, Y)$, so strictly speaking $\left(\phi^{\prime}, Z, \eta^{\prime}, g^{\prime}\right)$ is not a contact metric structure. Of course the difficulty is easily rectified and

$$
\eta=\frac{1}{2} \eta^{\prime}, Z_{\eta}=2 Z^{\prime}, \phi=\phi^{\prime}, g=\frac{1}{4} g^{\prime}
$$

is taken as the standard contact metric structure on $T_{1} M$.

Two simple examples are $E^{n+1} \times S^{n}(4)$, the tangent sphere bundle of Euclidean space, and $S^{3} \times S^{2}$ as the tangent sphere bundle of $S^{3}$ but not with the product metric; $S^{3} \times S^{2}$ with its standard contact metric structure is, however, a very nice example of a Sasakian Einstein manifold.

We remark that

$$
Z_{\eta}=2 v^{i}\left(\frac{\partial}{\partial x^{i}}\right)^{H}
$$

and that the vector field $v^{i}\left(\frac{\partial}{\partial x^{i}}\right)^{H}$ is the well-known geodesic flow on $T_{1} M$.

\subsection{Lie Groups}

We have already seen the Sasakian structures on the Heisenberg group and on the unit 3-sphere. We will see that the 3-sphere also carries non-Sasakian contact metric structures as we discuss Lie groups.

In a celebrated work, John Milnor [82] gave a complete classification of 3-dimensional Lie groups, their left invariant metrics and the relation of the structure constants to the Ricci curvature.

On a 3-dimensional Lie group $G$ we have a Lie algebra structure of the form

$$
\left[e_{2}, e_{3}\right]=c_{1} e_{1}, \quad\left[e_{3}, e_{1}\right]=c_{2} e_{2}, \quad\left[e_{1}, e_{2}\right]=c_{3} e_{3} .
$$

Define a Riemannian metric by $g\left(e_{i}, e_{j}\right)=\delta_{i j}$ at the identity and extend to all of $G$ by left translation. If some $c_{i}$ is non-zero, the dual 1-form $\omega_{i}$ is a contact form and the left invariant vector field determined by $e_{i}$ is its Reeb vector field. However, for the Riemannian metric $g$ to be an associated metric we must have $c_{i}=2$.

For example, the Lie group Sol, or equivalently the group $E(1,1)$ of rigid motions of the Minkowski plane, is topologically $\mathbb{R}^{3}$ and given by matrices of the form

$$
\left(\begin{array}{ccc}
e^{y} & 0 & z \\
0 & e^{-y} & x \\
0 & 0 & 1
\end{array}\right)
$$

The underlying Lie algebra structure is

$$
\left[e_{2}, e_{3}\right]=2 e_{1}, \quad\left[e_{3}, e_{1}\right]=-2 \lambda e_{2}, \quad\left[e_{1}, e_{2}\right]=0, \quad \lambda>0 .
$$

For the Lie algebra consider the three simple matrices

$$
\left(\begin{array}{lll}
0 & 0 & 1 \\
0 & 0 & 1 \\
0 & 0 & 0
\end{array}\right), \quad\left(\begin{array}{ccc}
0 & 0 & \frac{-1}{\sqrt{\lambda}} \\
0 & 0 & \frac{1}{\sqrt{\lambda}} \\
0 & 0 & 0
\end{array}\right), \quad\left(\begin{array}{ccc}
2 \sqrt{\lambda} & 0 & 0 \\
0 & -2 \sqrt{\lambda} & 0 \\
0 & 0 & 0
\end{array}\right)
$$


spanning it. However, let us use think in terms of the identification of the Lie algebra with $T_{I} E(1,1) \cong$ $\mathbb{R}^{3}(z, x, y)$ with basis

$$
\left(\begin{array}{l}
1 \\
1 \\
0
\end{array}\right), \quad\left(\begin{array}{c}
\frac{-1}{\sqrt{\lambda}} \\
\frac{1}{\sqrt{\lambda}} \\
0
\end{array}\right), \quad\left(\begin{array}{c}
0 \\
0 \\
2 \sqrt{\lambda}
\end{array}\right)
$$

Consider left translation by $\left(\begin{array}{ccc}e^{y} & 0 & z \\ 0 & e^{-y} & x \\ 0 & 0 & 1\end{array}\right)$ and its differential $\left(\begin{array}{ccc}e^{y} & 0 & 0 \\ 0 & e^{-y} & 0 \\ 0 & 0 & 1\end{array}\right)$; applying this to the basis vectors gives the vector fields

$$
Z_{1}=e^{-y} \frac{\partial}{\partial x}+e^{y} \frac{\partial}{\partial z}, \quad Z_{2}=\frac{1}{\sqrt{\lambda}}\left(e^{-y} \frac{\partial}{\partial x}-e^{y} \frac{\partial}{\partial z}\right), \quad Z_{3}=2 \sqrt{\lambda} \frac{\partial}{\partial y}
$$

whose Lie brackets satisfy the Lie algebra structure equations. With $Z_{1}$ as the Reeb vector field and $Z_{2}, Z_{3} \in$ $\mathcal{D}_{\eta}$, the contact form becomes

$$
\eta=\frac{1}{2}\left(e^{y} d x+e^{-y} d z\right)
$$

and the associated left invariant metric is

$$
g=\frac{1}{4}\left((1+\lambda) e^{2 y} d x^{2}+2(1-\lambda) d x d z+(1+\lambda) e^{-2 y} d z^{2}+\frac{1}{\lambda} d y^{2}\right) .
$$

This metric will appear again in Section $\mathrm{V}$ with $\lambda=1$.

The Lie group $S L(2, \mathbb{R})$, or its universal cover $\widetilde{S L}(2, \mathbb{R})$, has the above Lie algebra structure with two of the structure constants positive and one negative. Taking $\omega_{1}$ as the contact form we write the structure as

$$
\left[e_{2}, e_{3}\right]=2 e_{1}, \quad\left[e_{3}, e_{1}\right]=(1-\lambda) e_{2}, \quad\left[e_{1}, e_{2}\right]=(1+\lambda) e_{3}, \quad \lambda>1 .
$$

Similarly the Lie group $S U(2) \sim S^{3}$ has the above Lie algebra structure with all three structure constants positive. Again taking $\omega_{1}$ as the contact form the structure take the form

$$
\left[e_{2}, e_{3}\right]=2 e_{1}, \quad\left[e_{3}, e_{1}\right]=(1-\lambda) e_{2} \quad\left[e_{1}, e_{2}\right]=(1+\lambda) e_{3}
$$

where now $0 \leq \lambda<1$. Even for $\lambda=0$, which gives a Sasakian structure, this differs as an algebraic structure from the standard Lie algebra structure on $S^{3}$ where each structure constant is 2 . For $0<\lambda<1$ the structure is non-Sasakian.

Finally the Lie group $E(2)$ of rigid motions of the Euclidean plane is given by matrices of the form

$$
\left(\begin{array}{ccc}
\cos y & -\sin y & z \\
\sin y & \cos y & x \\
0 & 0 & 1
\end{array}\right)
$$

and the underlying Lie algebra structure is

$$
\left[e_{2}, e_{3}\right]=2 e_{1}, \quad\left[e_{3}, e_{1}\right]=0, \quad\left[e_{1}, e_{2}\right]=2 \lambda e_{3}, \quad \lambda>0 .
$$

To illustrate the relation of these Lie groups as contact metric manifolds to curvature, let us state a result of Domenico Perrone [92]. Denote by $\tau$ the scalar curvature and by

$$
W=\frac{1}{8}\left(\tau-\operatorname{Ric}\left(Z_{\eta}\right)+4\right)
$$

the Webster scalar curvature which we need not dwell on here.

Theorem 1. Let $\left(M^{3}, \eta, g\right)$ be a simply connected homogeneous contact metric manifold. Then $M$ is a Lie group $G$ and both $g$ and $\eta$ are left invariant. More precisely we have the following classification:

(1) If $G$ is unimodular, then it is one of the following Lie groups: 
1. The Heisenberg group when $W=\left|\$_{Z_{\eta}} g\right|=0$;

2. $S U(2)$ when $4 \sqrt{2} W>\left|\$_{Z_{\eta}} g\right|$;

3. the universal covering of the group of rigid motions of the Euclidean plane when $4 \sqrt{2} W=\left|\$_{Z_{\eta}} g\right|>0$;

4. the universal covering of $S L(2, \mathbb{R})$ when $-\left|\$_{Z_{\eta}} i g\right| \neq 4 \sqrt{2} W<\left|\$_{Z_{\eta}} g\right|$;

5. the group of rigid motions of the Minkowski plane when $4 \sqrt{2} W=-\left|\$_{Z_{\eta}} g\right|<0$.

(2) If $G$ is non-unimodular, its Lie algebra is given by

$$
\left[e_{1}, e_{2}\right]=\alpha e_{2}+2 Z_{\eta}, \quad\left[e_{1}, Z_{\eta}\right]=\gamma e_{2}, \quad\left[e_{2}, Z_{\eta}\right]=0,
$$

where $\alpha \neq 0, e_{1}, e_{2}=\phi e_{1} \in \mathcal{D}_{\eta}$ and $4 \sqrt{2} W<\left|\$_{Z_{\eta}} g\right|$. Moreover, if $\gamma=0$, the structure is Sasakian and $W=-\frac{\alpha^{2}}{4}$.

\subsection{Submanifolds}

Recall that a 1-dimensional integral submanifold of a contact manifold is a Legendre curve and we begin with an elementary property of Legendre curves in $\left(\mathbb{R}^{3}, \eta=d z-y d x\right)$. The projection $\gamma^{\star}$ of a closed Legendre curve $\gamma$ in $\mathbb{R}^{3}$ to the $x y$-plane must have self-intersections; moreover the algebraic (signed) area enclosed by $\gamma^{*}$ is zero. Since $d z-y d x=0$ along $\gamma$, this follows from the elementary formula for the area enclosed by a curve given by Green's theorem,

$$
0=-\int_{\gamma} d z=\int_{\gamma^{*}}-y d x=\text { area },
$$

the area being + for $\gamma^{\star}$ traversed counterclockwise and - for clockwise. Now one can think of the pair of $\gamma$ and its projection $\gamma^{*}$ in the following terms. Suppose that $\gamma$ itself does not have self-intersections and regard $\gamma^{\star}$ as a Lagrangian submanifold in $\mathbb{R}^{2} \cong \mathbb{C}$ with self-intersections; then think of going from $\gamma^{\star}$ to $\gamma$ as a way of removing the singularity but preserving the "Lagrangian-Legendre" property.

For example the map of the circle $u^{2}+v^{2}=1$ into $\mathbb{R}^{2}$ given by

$$
(u, v) \longrightarrow(v, 2 u v)
$$

has a double point, viz. $( \pm 1,0) \rightarrow(0,0)$. On the other hand the map of the circle $u^{2}+v^{2}=1$ into $\left(\mathbb{R}^{3}, \eta=\right.$ $d z-y d x)$ given by

$$
(u, v) \longrightarrow\left(2 u v, v, 2 u-\frac{4}{3} u^{3}\right)
$$

is an embedding and is a Legendre curve. This is the presentation in the literature; note the interchange of $v$ and $2 u v$, but $(0,0,0)$ is still above the double point $(0,0)$.

A well known result of Gromov [56] is that the sphere $S^{n}$ can not be embedded in $\mathbb{C}^{n}$ as a Lagrangian submanifold, so what is the next best thing? A generalization of the above example and an important Lagrangian submanifold of $\mathbb{R}^{2 n} \cong \mathbb{C}^{n}$ is the Whitney sphere. Let $\Omega=\sum_{i=1}^{n} d x^{i} \wedge d y^{i}$ be the standard symplectic form on $\mathbb{R}^{2 n}$ and consider the sphere $S^{n}$ in $\mathbb{R}^{n+1}$ given by $\sum_{i=0}^{n}\left(u^{i}\right)^{2}=1$ immersed in $\mathbb{R}^{2 n}$ by

$$
\left(u^{0}, \ldots, u^{n}\right) \longrightarrow\left(u^{1}, \ldots, u^{n}, 2 u^{0} u^{1}, \ldots, 2 u^{0} u^{n}\right) .
$$

Again notice the double point $( \pm 1,0, \ldots, 0)$ and it is easy to check that this immersed sphere is a Lagrangian submanifold of $\mathbb{R}^{2 n}$.

The Whitney sphere is often presented in another form, which, though slightly more complicated, lends itself to natural geometric characterization. For the Whitney sphere $M^{n}$ as a Lagrangian submanifold of $\mathbb{R}^{2 n} \cong$ $\mathbb{C}^{n}$, the immersion is

$$
\left(u^{0}, \ldots, u^{n}\right) \longrightarrow \frac{1}{1+\left(u^{0}\right)^{2}}\left(u^{1}, \ldots, u^{n}, u^{0} u^{1}, \ldots, u^{0} u^{n}\right) .
$$

This submanifold satisfies the relation

$$
|\mathbf{H}|^{2}=\frac{n+2}{n^{2}(n-1)} \tau
$$


where $\mathbf{H}$ is the mean curvature vector and $\tau$ the scalar curvature of $M^{n}$. This equality characterizes the Whitney sphere as a Lagrangian submanifold of $\mathbb{C}^{n}$. More precisely Borrelli, Chen and Morvan [24] proved that if $M^{n}$ is a Lagrangian submanifold of $\mathbb{C}^{n}$, then $|\mathbf{H}|^{2} \geq \frac{n+2}{n^{2}(n-1)} \tau$ with equality if and only if $M^{n}$ is either totally geodesic or a (piece of a) Whitney sphere.

Now embed $\sum_{i=0}^{n}\left(u^{i}\right)^{2}=1$ in the contact manifold $\mathbb{R}^{2 n+1}$ with its standard contact metric structure by

$$
\left(u^{0}, \ldots, u^{n}\right) \longrightarrow\left(2 u^{0} u^{1}, \ldots, 2 u^{0} u^{n}, u^{1}, \ldots, u^{n}, 2 u^{0}-\frac{4}{3}\left(u^{0}\right)^{3}\right)
$$

giving an embedded sphere as an integral submanifold of the standard contact structure. We refer to this sphere as a contact Whitney sphere.

In the contact manifold $\mathbb{R}^{2 n+1}$ with its standard contact metric structure we also have a second presentation of the contact Whitney sphere as an embedded sphere and an integral submanifold of the contact structure, namely

$$
\left(u^{0}, \ldots, u^{n}\right) \longrightarrow \frac{1}{1+\left(u^{0}\right)^{2}}\left(u^{0} u^{1}, \ldots, u^{0} u^{n}, u^{1}, \ldots, u^{n}, \frac{u^{0}}{1+\left(u^{0}\right)^{2}}\right)
$$

which can be viewed as the lift of the Whitney sphere in $\mathbb{C}^{n}$. For an integral submanifold $M^{n}$ of $\mathbb{R}^{2 n+1}$ we also have $|\mathbf{H}|^{2} \geq \frac{n+2}{n^{2}(n-1)} \tau$ with equality if and only if $M^{n}$ is either totally geodesic or a (piece of a) contact Whitney sphere (Carriazo and B. [11]). Note the segment of the $z$-axis or chord joining the points $\left(0, \ldots, 0, \pm \frac{1}{4}\right)$ (or $\left(0, \ldots, 0, \pm \frac{2}{3}\right)$ in the first presentation). This reminds us of the Arnold Chord Conjecture [2] concerning integral curves of the Reeb vector field joining points of a Legendre submanifold. More precisely, Arnold conjectured that every closed Legendre curve in $S^{3}$ with its standard contact subbundle has a Reeb chord for any contact form defining it. This was proved by Mohnke [84] for spheres $S^{2 n+1}$ with their standard contact subbundles. More recently Hutchings and Taubes [63],[64] have extended and proved the conjecture for general contact 3-manifolds.

Before going further let us present briefly the notation of submanifold theory. For a submanifold $M$ of a Riemannian manifold $(\tilde{M}, \tilde{g})$ we denote the induced metric by $g$. Then the Levi-Civita connection $\nabla$ of $g$ and the second fundamental form $\sigma$ are related to the ambient Levi-Civita connection $\tilde{\nabla}$ by

$$
\tilde{\nabla}_{X} Y=\nabla_{X} Y+\sigma(X, Y) .
$$

For a normal vector field $v$ we denote by $A_{v}$ the corresponding Weingarten map and we denote by $\nabla^{\perp}$ the connection in the normal bundle; in particular $A_{v}$ and $\nabla^{\perp}$ are defined by

$$
\tilde{\nabla}_{X} v=-A_{v} X+\nabla_{X}^{\frac{1}{X}} v
$$

The Gauss equation is

$$
\tilde{R}(X, Y, Z, W)=R(X, Y, Z, W)+\tilde{g}(\sigma(X, Z), \sigma(Y, W))-\tilde{g}(\sigma(Y, Z), \sigma(X, W)) .
$$

Defining the covariant derivative of $\sigma$ by $\left(\nabla^{\prime} \sigma\right)(X, Y, Z)=\nabla_{X}^{\frac{1}{X}} \sigma(Y, Z)-\sigma\left(\nabla_{X} Y, Z\right)-\sigma\left(Y, \nabla_{X} Z\right)$ the Codazzi equation is

$$
\left(\tilde{R}_{X Y} Z\right)^{\perp}=\left(\nabla^{\prime} \sigma\right)(X, Y, Z)-\left(\nabla^{\prime} \sigma\right)(Y, X, Z) .
$$

Finally for normal vector fields $v$ and $\zeta$ the equation of Ricci-Kühne is

$$
\tilde{R}(X, Y, v, \zeta)=R^{\perp}(X, Y, v, \zeta)-g\left(\left[A_{\nu}, A_{\zeta}\right] X, Y\right) .
$$

Again recall that a submanifold $M^{r}$ of $M^{2 n+1}$ is an integral submanifold if $\eta(X)=0$ for every tangent vector $X$. It is clear then that for any pair of tangent vector fields we have

$$
d \eta(X, Y)=\frac{1}{2}(X \eta(Y)-Y \eta(X)-\eta([X, Y]))=0 .
$$


Thus in terms of associated metrics, $g(X, \phi Y)=0$, in particular $\phi$ maps tangent vectors to normal vectors; also since $Z_{\eta}$ is a normal vector, the dimension $r$ can be at most $n$. On the other hand by the Darboux theorem we have local coordinates $\left(x^{1}, \ldots, x^{n}, y^{1}, \ldots, y^{n}, z\right)$ with respect to which $\eta=d z-\sum_{i=1}^{n} y^{i} d x^{i}$. Therefore $x^{i}=$ const., $z=$ const. define an $n$-dimensional integral submanifold. Since $g(X, \phi Y)=0$ integral submanifolds are sometimes called $C$-totally real submanifolds and if the integral submanifold has dimension $n$, it is sometimes called a Legendre (or Legendrian) submanifold in analogy with Lagrangian submanifolds in symplectic geometry.

Turning to specific results we first mention the following result of Van Lindt, Verheyen and Verstraelen [111] because of the nice technique used in the proof which is due to A. Ros [97].

Theorem 2. Let $M^{n}$ be a compact integral submanifold minimally immersed in a Sasakian space form $\left(\tilde{M}^{2 n+1}(c), \tilde{\phi}, \tilde{Z}_{\tilde{\eta}}, \tilde{\eta}, \tilde{g}\right)$ with $c>-3$. If the sectional curvature $K$ of $M^{n}$ is positive, then it is totally geodesic.

The idea is to define a real-valued function $f$ on the unit tangent bundle $T_{1} M^{n}$ by

$$
f(V)=\tilde{g}(\sigma(V, V), \tilde{\phi} V) .
$$

Since $T_{1} M^{n}$ is compact, $f$ attains its maximum at a unit tangent vector $V$ at some point $p \in M^{n}$. Then for any tangent vector $U$ at $p$, let $\gamma(t)$ be the geodesic in $M^{n}$ with $\gamma(0)=p$ and $\gamma^{\prime}(0)=U$. Let $V(t)$ be the parallel translate of $V$ along $\gamma$. Then

$$
0=\left.\frac{d}{d t} f(V(t))\right|_{t=0} \text { and } 0 \geq\left.\frac{d^{2}}{d t^{2}} f(V(t))\right|_{t=0} .
$$

The analysis of these relations using the equations of Gauss, Codazzi and Ricci-Kühne is quite involved but interesting and gives the result.

If the sectional curvature is only $\geq 0$, one can do better in dimension 7 , namely, we have the following result of Dillen and Vrancken [37].

Theorem 3. Let $M^{3}$ be a compact integral submanifold of the standard Sasakian structure on $S^{7}(1)$ which is minimally immersed. If $K \geq 0$, then either $M^{3}$ is totally geodesic, $M^{3}$ is a covering of the 3-torus or $M^{3}$ is a covering of $S^{1}\left(\frac{1}{\sqrt{3}}\right) \times S^{2}\left(\frac{4}{3}\right)$.

In this theorem $0 \leq K \leq \frac{4}{3}$ and both extreme values are attained in the last case. On the other hand, note the later result of Dillen and Vrancken [38].

Theorem 4. If $M^{n}$ is a compact minimal integral submanifold of $S^{2 n+1}(1)$ and if $0 \leq K \leq 1$, then $K$ is identically 0 or 1.

There are many other results along these lines in the literature. There are also many results concerning invariant submanifolds which we do not have time to discuss, so we only give the definition and a few remarks. For a contact metric manifold $\tilde{M}^{2 n+1}$ with structure tensors $\left(\tilde{\phi}, \tilde{Z}_{\eta}, \tilde{\eta}, \tilde{g}\right)$ a submanifold $M$ is said to be invariant if $\tilde{\phi} T_{p} M \subset T_{p} M$. Some authors also require that $\tilde{Z}_{\eta}$ be tangent to $M$ but this is a consequence. An invariant submanifold inherits a contact metric structure by restriction. Moreover for the induced structure $\left(\phi, Z_{\eta}, \eta, g\right)$ we have $h=\left.\tilde{h}\right|_{M}$ as well. Also for the second fundamental form we have

$$
\sigma\left(Z_{\eta}, X\right)=\tilde{\nabla}_{X} \tilde{Z}_{\eta}-\nabla_{X} Z_{\eta}=-\tilde{\phi} X-\tilde{\phi} \tilde{h} X-(-\phi X-\phi h X)=0 .
$$

A result of Chinea [30] and independently of Endo [41] is the following, similar to the fact that an invariant submanifold of a Kähler manifold is minimal.

Theorem 5. An invariant submanifold of a contact metric manifold is minimal. 
It is also known and not difficult to prove that an invariant submanifold of a K-contact (resp. Sasakian) manifold is K-contact (resp. Sasakian). Moreover, invariant submanifolds respect the Boothby-Wang fibration; in particular we have the following result of Harada [58].

Theorem 6. Let $M$ be a compact invariant submanifold of a compact regular Sasakian manifold $\tilde{M}$. Then $M$ is regular and $M / Z_{\eta}$ is a Kähler (invariant) submanifold of $\tilde{M} / Z_{\eta}$.

For example consider the complex quadric $Q^{n-1}$ in $\mathbb{C} P^{n}$ together with the Hopf fibration $S^{2 n+1} \longrightarrow \mathbb{C} P^{n}$. Then the set of fibres over $Q^{n-1}$ form a codimension 2 invariant submanifold of the Sasakian structure on $S^{2 n+1}$.

\section{Curvature of Contact Metric Manifolds}

In this section we discuss several aspects of the curvature of contact metric manifolds. Let $M^{2 n+1}$ be a contact metric manifold with structure tensors $\left(\phi, Z_{\eta}, \eta, g\right)$ and recall the important tensor field $h=\frac{1}{2} \$_{Z_{\eta}} \phi$. We have already noted that

$$
\nabla_{X} Z_{\eta}=-\phi X-\phi h X
$$

Differentiating this with respect to $Z_{\eta}$ we can compute $R_{Z_{\eta} X} Z_{\eta}$. This yields the following formulas

$$
\begin{gathered}
\left(\nabla_{Z_{\eta}} h\right) X=\phi X-h^{2} \phi X-\phi R_{X Z_{\eta}} Z_{\eta}, \\
\frac{1}{2}\left(R_{X Z_{\eta}} Z_{\eta}-\phi R_{\phi X} Z_{\eta} Z_{\eta}\right)=-\phi^{2} X-h^{2} X .
\end{gathered}
$$

From the second formula one has as an easy corollary

$$
\operatorname{Ric}\left(Z_{\eta}\right)=2 n-\operatorname{tr} h^{2}
$$

and we see immediately that a contact metric manifold is K-contact if and only if

$$
\operatorname{Ric}\left(Z_{\eta}\right)=2 n \text {. }
$$

One also sees from these formulas that a contact metric manifold is K-contact if and only if the sectional curvature of all plane sections containing $Z_{\eta}$ are equal to +1 . We remarked earlier that the Sasakian condition can be written as,

$$
\left(\nabla_{X} \phi\right) Y=g(X, Y) Z_{\eta}-\eta(Y) X, \text { and as, } R_{X Y} Z_{\eta}=\eta(Y) X-\eta(X) Y \text {. }
$$

An early result (B. [6]) is that in dimension $\geq 5$ there are no flat associated metrics. This was generalized by Olszak [90] to the following.

Theorem 1. If a contact metric manifold $M^{2 n+1}$ is of constant curvature $c$ and dimension $\geq 5$, then $c=+1$ and the structure is Sasakian. In dimension 3, constant curvature arises only for $c=0,1$ and again the latter case is Sasakian.

I conjecture that the proper generalization of non-flatness is that, aside from the flat 3-dimen-sional case, a contact metric manifold should have some positive curvature. Strictly speaking, in dimension 3 this is false by virtue of an example due to Krouglov [77] of a 3-dimensional contact metric manifold which is negatively curved on some neighborhood as we will see below, but which is not globally of non-positive curvature. Let us first however give a result of Rukimbira [98] based on work of Zeghib [117]. If the manifold is compact and we ask for strictly negative curvature we can answer this question in the negative using the following deep result of A. Zeghib on geodesic plane fields. Recall that a $k$-dimensional plane field on an $n$-dimensional Riemannian manifold is said to be geodesic if any geodesic tangent to the plane field at one point is tangent to it at every point. Zeghib's result is the following. 
Theorem 2. A compact negatively curved Riemannian manifold has no $C^{1}$ geodesic plane field.

Since for any contact metric structure the integral curves of $Z_{\eta}$ are geodesics, $Z_{\eta}$ determines a geodesic line field to which we can apply the theorem of Zeghib as was pointed out by Rukimbira. Thus we have the following theorem.

Theorem 3. On a compact contact manifold, there is no associated metric of strictly negative curvature.

The fact that hyperbolic space has many 1-dimensional totally geodesic foliations, does not violate the positive curvature conjecture, since the hyperbolic metric cannot be an associated metric of any contact structure by the above theorem of Olszak.

A contact manifold is said to be homogenous if it admits a transitive Lie group of diffeomorphisms which preserving the contact form. A contact metric manifold is said to be homogenous if it admits a transitive Lie group of diffeomorphisms which preserves the structure tensors $\left(\phi, Z_{\eta}, \eta, g\right)$. We now have the following results of A. Lotta [79].

Theorem 4. In dimensions $\geq 5$ there are no homogeneous, simply connected contact manifolds which admit a Riemannian metric of non-positive curvature and for which the Reeb vector field is orthogonal to the contact sub-bundle.

Theorem 5. Let $M^{2 n+1}$ be a homogeneous, simply connected contact metric manifold of non-positive curvature. Then the manifold is 3-dimensional, flat and equivalent to the universal cover of $E(2)$ with a left invariant contact metric structure.

In [77] V. Krouglov gave the following example of a contact metric structure on $\mathbb{R}^{3}$ which we present with a few notational modifications. Consider the standard Darboux form $\eta=\frac{1}{2}(d z-y d x)$ and its Reeb vector field $Z_{\eta}=2 \frac{\partial}{\partial z}$. The associated metric that he chose is essentially

$$
g=\frac{1}{4}\left(\begin{array}{ccc}
\sqrt{2} e^{-z}+y^{2} & -1 & -y \\
-1 & \sqrt{2} e^{z} & 0 \\
-y & 0 & 1
\end{array}\right)
$$

The vector fields

$$
X=\frac{\sqrt{2} e^{z / 2}}{\sqrt{\sqrt{2}-1}}\left(\begin{array}{c}
1 \\
e^{-z} \\
y
\end{array}\right), \quad Y=\frac{\sqrt{2} e^{z / 2}}{\sqrt{\sqrt{2}+1}}\left(\begin{array}{c}
1 \\
-e^{-z} \\
y
\end{array}\right)
$$

together with $Z_{\eta}$ form an orthonormal basis. Now let

$$
U=\alpha X+\beta Y+\gamma Z_{\eta}, \quad V=\lambda X+\mu Y+v Z_{\eta}
$$

be two independent vector fields. Then by direct computation

$$
\begin{gathered}
R(U, V, V, U)=-(\alpha \mu-\beta \lambda)^{2}\left(1+2 \sqrt{2} y^{2} e^{z}\right)-(\alpha \nu-\gamma \lambda)^{2}-(\beta \nu-\gamma \mu)^{2} \\
-4 \sqrt{1+\sqrt{2}}(\alpha \mu-\beta \lambda)(\alpha \nu-\gamma \lambda) y e^{z / 2}+\frac{4}{\sqrt{1+\sqrt{2}}}(\alpha \mu-\beta \lambda)(\beta \nu-\gamma \mu) y e^{z / 2}
\end{gathered}
$$

which is negative at the origin and therefore negative on a neighborhood of the origin. This neighborhood would then be a contact metric manifold of negative curvature even though it is not complete. For $\alpha=\mu=$ $v=1$ and $\beta=\gamma=\lambda=0$

$$
R\left(X, Y+Z_{\eta}, Y+Z_{\eta}, X\right)=-\left(1+2 \sqrt{2} y^{2} e^{z}\right)-1-4 \sqrt{1+\sqrt{2}} y e^{z / 2} .
$$

At $y=-1, z=0$ this is positive, so $\mathbb{R}^{3}$ with the standard Darboux form and this metric has some positive curvature. 
There is a more general class of contact metric manifolds than the Sasakian ones, called $(\kappa, \mu)$-manifolds which has turned out to be of more interest than one might have originally anticipated. Let us begin with the following theorem (B. [7]).

Theorem 6. A contact metric manifold $M^{2 n+1}$ satisfying $R_{X Y} Z_{\eta}=0$ is locally isometric to $E^{n+1} \times S^{n}(4)$ for $n>1$ and flat for $n=1$.

The structure is the standard contact metric structure as described earlier on the tangent sphere bundle of Euclidean space, $E^{n+1} \times S^{2}(4)$.

Themis Koufogiorgos observed that the condition $R_{X Y} Z_{\eta}=0$ is not $\mathcal{D}$-homothetic invariant unlike a number of other conditions we have mentioned, rather it takes the form

$$
R_{X Y} Z_{\eta}=\kappa(\eta(Y) X-\eta(X) Y)+\mu(\eta(Y) h X-\eta(X) h Y)
$$

for constants $\kappa$ and $\mu$. This form, however, is $\mathcal{D}$-homothetic invariant, that is, for the deformed metric $\bar{g}=$ $a g+a(a-1) \eta \otimes \eta, \bar{R}_{X Y} \bar{Z}_{\eta}$ takes this form with

$$
\bar{\kappa}=\frac{\kappa+a^{2}-1}{a^{2}}, \quad \bar{\mu}=\frac{\mu+2 a-2}{a} .
$$

A contact metric manifold satisfying $\left({ }^{\star}\right)$ is called a $(\kappa, \mu)$-manifold as introduced and developed as an interesting class of contact metric manifolds by Koufogiorgos, Papatoniou, and the author in [14].

It is known that $\kappa$ is always $\leq 1$ and if $\kappa=1$, the structure is Sasakian. Moreover if $\kappa<1$, the $(\kappa, \mu)$ condition determines the curvature of $M^{2 n+1}$ completely. Also a $(\kappa, \mu)$-manifold is a strongly pseudoconvex CR-manifold.

Furthermore, if $\kappa<1$, the non-zero eigenvalues of $h$ are $\pm \sqrt{1-\kappa}$ each with multiplicty $n$. Let $\lambda$ be the positive eigenvalue. Then $M^{2 n+1}$ admits three mutually orthogonal subbundles $\mathcal{D}(0), \mathcal{D}(\lambda)$ and $\mathcal{D}(-\lambda)$ which are integrable. The subbundles $\mathcal{D}(\lambda)$ and $\mathcal{D}(-\lambda)$ were further studied as Legendre foliations by Cappelletti Montano and Di Terlizzi [27].

For a non-Sasakian $(\kappa, \mu)$-manifold $M$, Boeckx [16] introduced an invariant

$$
I_{M}=\frac{1-\frac{\mu}{2}}{\sqrt{1-\kappa}}
$$

and showed that for two non-Sasakian $(\kappa, \mu)$-manifolds $\left(M_{i}, \phi_{i}, \xi_{i}, \eta_{i}, g_{i}\right), i=1,2$, we have $I_{M_{1}}=I_{M_{2}}$ if and only if up to a $\mathcal{D}$-homothetic deformation, the two spaces are locally isometric as contact metric manifolds. Thus we know all non-Sasakian $(\kappa, \mu)$-manifolds locally as soon as we have for every odd dimension $2 n+1$ and for every possible value of the invariant $I$, one $(\kappa, \mu)$-manifold $(M, \phi, \xi, \eta, g)$ with $I_{M}=I$. The standard contact metric structure on the tangent sphere bundle of a manifold of constant curvature $c \neq 1, I=\frac{1+c}{|1-c|}$. Therefore as $c$ varies, $I$ takes on every value $>-1$. Boeckx now gives an example for any odd dimension and value of $I \leq-1$; his construction is as follows.

Let $\mathfrak{g}$ be a $(2 n+1)$-dimensional Lie algebra, $n \geq 2$. Introduce a basis for $\mathfrak{g}$,

$$
\left\{Z, X_{1}, \ldots, X_{n}, Y_{1}, \ldots, Y_{n}\right\},
$$

and for real numbers $\alpha$ and $\beta$ define the Lie bracket by

$$
\begin{gathered}
{\left[Z, X_{1}\right]=-\frac{\alpha \beta}{2} X_{2}-\frac{\alpha^{2}}{2} Y_{1}, \quad\left[Z, X_{2}\right]=\frac{\alpha \beta}{2} X_{1}-\frac{\alpha^{2}}{2} Y_{2}, \quad\left[Z, X_{i}\right]=-\frac{\alpha^{2}}{2} Y_{i}, i \geq 3,} \\
{\left[Z, Y_{1}\right]=\frac{\beta^{2}}{2} X_{1}-\frac{\alpha \beta}{2} Y_{2}, \quad\left[Z, Y_{2}\right]=\frac{\beta^{2}}{2} X_{2}+\frac{\alpha \beta}{2} Y_{1}, \quad\left[Z, Y_{i}\right]=\frac{\beta^{2}}{2} X_{i}, i \geq 3,} \\
{\left[X_{1}, X_{i}\right]=\alpha X_{i}, i \neq 1, \quad\left[X_{i}, X_{j}\right]=0, i, j \neq 1,} \\
{\left[Y_{2}, Y_{i}\right]=\beta Y_{i}, i \neq 2, \quad\left[Y_{i}, Y_{j}\right]=0, i, j \neq 2,} \\
{\left[X_{1}, Y_{1}\right]=-\beta X_{2}+2 Z, \quad\left[X_{1}, Y_{i}\right]=0, i \geq 2,}
\end{gathered}
$$




$$
\begin{gathered}
{\left[X_{2}, Y_{1}\right]=\beta X_{1}-\alpha Y_{2}, \quad\left[X_{2}, Y_{2}\right]=\alpha Y_{1}+2 Z, \quad\left[X_{2}, Y_{i}\right]=\beta X_{i}, i \geq 3,} \\
{\left[X_{i}, Y_{1}\right]=-\alpha Y_{i}, i \geq 3, \quad\left[X_{i}, Y_{2}\right]=0, i \geq 3,} \\
{\left[X_{i}, Y_{j}\right]=\delta_{i j}\left(-\beta X_{2}+\alpha Y_{1}+2 Z\right), i, j \geq 3 .}
\end{gathered}
$$

The associated Lie group $G$ is not unimodular if not both $\alpha$ and $\beta$ are equal to zero. Now define a metric on $G$ by left translation of the basis $\left\{Z, X_{1}, \ldots, X_{n}, Y_{1}, \ldots, Y_{n}\right\}$, taken as orthonormal at the identity. Then taking $\eta$ as the metric dual of $Z$ and defining $\phi$ by $\phi Z=0, \phi X_{i}=Y_{i}$ and $\phi Y_{i}=-X_{i}$, we have a contact metric structure on $G$. Now for the present purpose suppose that $\beta^{2}>\alpha^{2} . G$ is a non-Sasakian $(\kappa, \mu)$-manifold and

$$
I_{G}=-\frac{\beta^{2}+\alpha^{2}}{\beta^{2}-\alpha^{2}} \leq-1 ;
$$

thus for appropriate choices of $\beta>\alpha \geq 0, I_{G}$ attains any value $\leq-1$.

For the 3-dimensional case, consider the Lie algebra

$$
[Z, X]=-\frac{\alpha^{2}}{2} Y, \quad[Z, Y]=\frac{\beta^{2}}{2} X, \quad[X, Y]=2 Z
$$

which corresponds to a unimodular Lie group. Boeckx points out that for appropriate values of $\alpha$ and $\beta$ we obtain left invariant contact metric structures with values of the invariant $I_{G}$ as follows: $I_{S U(2)}>1, I_{E(2)}=1$, $-1<I_{S L(2, \mathbb{R})}<1, I_{E(1,1)}=-1$ and $I_{S L(2, \mathbb{R})}<-1$.

The Lie group of rigid motions of the Minkowski plane, $E(1,1)$, carries a $(0,4)$-structure and will be discussed further in Section V.

In [76] Koufogiorgos and Tsichlias considered the question of contact metric manifolds for which $Z_{\eta}$ satisfies the $(\kappa, \mu)$ condition but where $\kappa$ and $\mu$ are functions rather than constants and called these spaces generalized $(\kappa, \mu)$-manifolds. They showed that in dimensions $\geq 5, \kappa$ and $\mu$ must be constant and in dimension 3 gave an example where $\kappa$ and $\mu$ are not constants. Moreover this idea is closely related to the question of the Reeb vector field as a map into the tangent sphere bundle being a harmonic map.

Next let us discuss the notion of $\phi$-sectional curvature. This idea plays the role in Sasakian geometry that holomorphic sectional curvature plays in Kähler geometry. A plane section in $T_{m} M^{2 n+1}$ is called a $\phi$-section if there exists a vector $X \in T_{m} M^{2 n+1}$ orthogonal to $Z_{\eta}$ such that $\{X, \phi X\}$ span the section. The sectional curvature $K(X, \phi X)$ is called $\phi$-sectional curvature.

Recall that the sectional curvatures of a Riemannian manifold determine the curvature transformation $R_{X Y} Z$. It is also well known that the holomorphic sectional curvatures of a Kähler manifold determine the curvature completely. Moskal [85] showed that on a Sasakian manifold the $\phi$-sectional curvatures determine the curvature completely. Ogiue [88] proved the following result giving rise to the concept of a Sasakian space form.

Theorem 7. If the $\phi$-sectional curvature at any point of a Sasakian manifold of dimension $\geq 5$ is independent of the choice of $\phi$-section at the point, then it is constant on the manifold and the curvature tensor is given by

$$
\begin{gathered}
R_{X Y} Z=\frac{c+3}{4}(g(Y, Z) X-g(X, Z) Y) \\
+\frac{c-1}{4}\left(\eta(X) \eta(Z) Y-\eta(Y) \eta(Z) X+g(X, Z) \eta(Y) Z_{\eta}-g(Y, Z) \eta(X) Z_{\eta}\right. \\
+d \eta(Z, Y) \phi X-d \eta(Z, X) \phi Y+2 d \eta(X, Y) \phi Z)
\end{gathered}
$$

where $c$ is the constant $\phi$-sectional curvature.

A $\mathcal{D}$-homothetic deformation of a Sasakian space form is again a Sasakian space form. For example, the odddimensional unit sphere with its standard Sasakian structure can be deformed to a Sasakian space form of constant $\phi$-secitonal curvature $c=\frac{4}{a}-3$ giving any $c>-3$. In Section I we briefly mentioned the Sasakian 
space form $\mathbb{R}^{2 n+1}(-3)$. If $B^{n}$ is a simply connected, bounded domain in $\mathbb{C}^{n}, B^{n} \times \mathbb{R}$ has a Sasakian structure of constant $\phi$-sectional curvature $<-3$.

Th. Koufogiorgos [75] studied $(\kappa, \mu)$-manifolds of dimension $\geq 5$ for which the $\phi$-sectional curvature at any point is independent of the choice of $\phi$-section at the point. He proved that the $\phi$-sectional curvature is constant and obtained the curvature tensor explicitly.

In the general context of contact metric manifolds J. T. Cho [31] introduced the notion of a contact Riemannian space form. We get at this notion in the following way. Mitric [83] and Tanno [109] showed hat the tangent sphere bundle with its standard contact metric structure is a CR-manifold if and only if the base manifold is of constant curvature. Cho first computes the covariant derivative of $h$ in this case obtaining

$$
\left(\nabla_{X} h\right) Y=g\left(\left(h-h^{2}\right) \phi X, Y\right) \xi+\eta(Y)\left(h-h^{2}\right) \phi X-\mu \eta(X) h \phi Y
$$

where $\mu$ is a constant. He then abstracts this idea and defines the class $\mathfrak{Q}$ of contact metric CR-manifolds for which the covariant derivative of $h$ satisfies the above condition. We remark that in the study of contact metric manifolds in general, lack of control of the covariant derivative of $h$ is often an obstacle to further results, so this is a welcome consideration.

Now for a contact metric manifold $M^{2 n+1}$ with $n>1$ in the class $\mathfrak{Q}$ for which the $\phi$-sectional curvature is independent of the choice of $\phi$-section, Cho shows that the $\phi$-sectional curvature is constant on $M^{2 n+1}$ and computes the curvature tensor explicitly. He then defines a contact Riemannian space form to be a complete, simply connected contact metric manifold of class $\mathfrak{Q}$ of constant $\phi$-sectional curvature. Cho also gave a number of non-Sasakian examples and showed that a contact Riemannian space form is locally homogeneous and is strongly locally $\phi$-symmetric, a notion that we will discuss shortly.

Another natural curvature question to ask is when might a contact metric manifold be locally symmetric. In 1962 Okumura [89] showed that a locally symmetric Sasakian manifold is locally isometric to $S^{2 n+1}(1)$ and it took until 2006 before Boeckx and Cho [20] could show that a locally symmetric contact metric manifold is locally isometric to $S^{2 n+1}(1)$ or $E^{n+1} \times S^{n}(4)$. Recall that the latter structure is that of the contact metric structure on the tangent sphere bundle of Euclidean space. In particular, the product metric on $S^{3} \times S^{2}$ is not an associated metric; $S^{3} \times S^{2}$ does, however, carry a Sasakian Einstein structure (Tanno [107]). Perrone and Vanhecke [95] proved that the only 5-dimensional compact, simply connected, homogeneous contact manifolds are diffeomorphic to $S^{5}$ or $S^{3} \times S^{2}$.

These results can be regarded as saying that the idea of being locally symmetric is too strong. This was already recognized in the Sasakian case by T. Takahashi [105] and he introduced the notion of a locally $\phi$ symmetric space. A Sasakian manifold is said to be a Sasakian locally $\phi$-symmetric space if

$$
\phi^{2}\left(\nabla_{V} R\right)_{X Y} Z=0
$$

for all vector fields $V, X, Y, Z$ orthogonal to $Z_{\eta}$. It is easy to check that Sasakian space forms are locally $\phi$ symmetric spaces.

Note that on a Sasakian manifold $M$, or more generally on a K-contact manifold, a geodesic that is initially orthogonal to $Z_{\eta}$ remains orthogonal to $Z_{\eta}$. We call such a geodesic a $\phi$-geodesic. A local diffeomorphism $s_{m}$ of $M, m \in M$, is a $\phi$-geodesic symmetry if its domain contains a (possibly) smaller domain $\mathcal{U}$ such that for every $\phi$-geodesic $\gamma(s)$ parametrized by arc length such that $\gamma(0)$ is in the intersection of $\mathcal{U}$ and the integral curve of $Z_{\eta}$ through $m$ and

$$
\left(s_{m} \circ \gamma\right)(s)=\gamma(-s)
$$

for all $s$ with $\gamma( \pm s) \in \mathcal{U}$. 


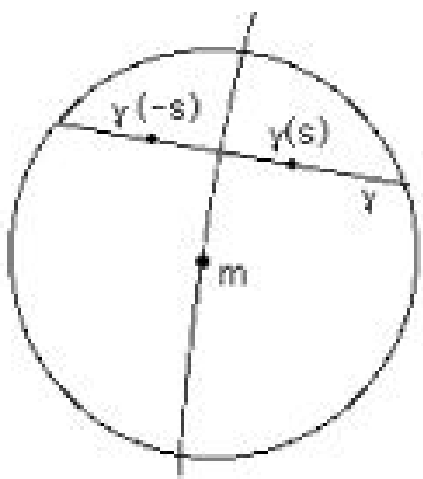

Takahashi defines a Sasakian manifold to be a Sasakian globally $\phi$-symmetric space by requiring that any $\phi$-geodesic symmetry can be extended to a global automorphism of the structure and that the Killing vector field $Z_{\eta}$ generates a 1-parameter group of global transformations. Among the main results of Takahashi are the following three theorems.

Theorem 8. A Sasakian locally $\phi$-symmetric space is locally isometric to a Sasakian globally $\phi$-symmetric space and a complete, connected, simply-connected Sasakian locally $\phi$-symmetric space is globally $\phi$ symmetric.

Theorem 9. A Sasakian manifold is locally $\phi$-symmetric if and only if it admits a $\phi$-geodesic symmetry at every point which is a local automorphism of the structure.

Now suppose that $U$ is a neighborhood on $M$ on which $Z_{\eta}$ is regular, then since $M$ is Sasakian, the projection $\pi: \mathcal{U} \longrightarrow \mathcal{V}=\mathcal{U} / Z_{\eta}$ gives a Kähler structure on $\mathcal{V}$. Furthermore if $\underline{\mathrm{s}}_{\pi(m)}$ denotes the geodesic symmetry on $\mathcal{V}$ at $\pi(m)$, then $\underline{\mathrm{s}}_{\pi(m)} \circ \pi=\pi \circ S_{m}$.

Theorem 10. A Sasakian manifold is locally $\phi$-symmetric if and only if each Kähler manifold which is the base of a local fibering is a Hermitian locally symmetric space.

In the spirit of the fact that a Riemannian manifold is locally symmetric if and only if the local geodesic symmetries are isometries and in view of the above results of Takahashi, we state the following extension due to L. Vanhecke and his school in the late 1980s.

Theorem 11. On a Sasakian locally $\phi$-symmetric space, local $\phi$-geodesic symmetries are isometries. Conversely if on a K-contact manifold the local $\phi$-geodesic symmetries are isometries, the manifold is a Sasakian locally $\phi$-symmetric space.

Without the K-contact property one loses the fact that a geodesic, initially orthogonal to $Z_{\eta}$ remains orthogonal to $Z_{\eta}$ and until the late 1990s it was not clear what local $\phi$-symmetry should mean for a general contact metric manifold. We have seen that in the Sasakian case local $\phi$-symmetry is equivalent to reflections in the integral curves of the Reeb vector field being isometries. In [19] Boeckx, Bueken and Vanhecke formalized two notions. A contact metric manifold is a weakly locally $\phi$-symmetric space if it satisfies $\phi^{2}\left(\nabla_{V} R\right)_{X Y} Z=0$ for all vector fields $V, X, Y, Z$ orthogonal to $Z_{\eta}$ as in the Sasakian case. A contact metric manifold is a strongly locally $\phi$-symmetric space if reflections in the integral curves of the Reeb vector field are isometries.

Calvaruso, Perrone and Vanhecke [26] showed that in dimensional 3, a strongly locally $\phi$-symmetric space is either K-contact with constant scalar curvature or is a $(\kappa, \mu)$-manifold with $\kappa<1$. They also showed that a 3-dimensional contact metric manifold is a strongly locally $\phi$-symmetric space if and only if it is locally contact homogeneous i.e. the pseudo-group of local automorphisms of the contact metric structure acts transitively on the manifold, and $Z_{\eta}$ is an eigenvector of the Ricci operator. Boeckx also showed [16] that a nonSasakian $(\kappa, \mu)$-manifold is strongly locally $\phi$-symmetric and also locally contact homogeneous. Conversely 
in [17] Boeckx showed that a strongly locally $\phi$-symmetric, locally contact homogeneous contact metric manifold must be a $(\kappa, \mu)$-manifold.

Examples of strongly locally $\phi$-symmetric spaces include the non-Sasakian $(\kappa, \mu)$-manifolds. Special cases of these are the non-abelian 3-dimensional unimodular Lie groups with left-invariant contact metric structures. Boeckx, Bueken and Vanhecke [19] also gave an example of a non-unimodular Lie group with a weakly locally $\phi$-symmetric contact metric structure which is not strongly locally $\phi$-symmetric.

Finally there is the matter of conformally flat contact metric manifolds, a topic on which little is known. In dimension 3 there exist conformally flat contact metric manifolds which are not of constant curvature. However, in dimensions $\geq 5$ it is an open question as to whether there exist conformally flat contact metric manifolds other than the constant curvature +1 , Sasakian case. A discuss of this and other conformally flat questions can be found in the essay by K. Bang and the author [3].

\section{Complex Contact Manifolds and Some Older Style Topology}

\subsection{Complex Contact Manifolds}

The study of complex contact manifolds is almost as old as the modern theory of real contact manifolds, that is, just shortly after the Boothby-Wang fibration. In particular, this study begins with the work of Kobayashi [70] and Boothby [21], [22]. Shortly thereafter J. A. Wolf [112] studied homogeneous complex contact manifolds. Here we will give a brief introduction to this subject from a geometric point of view.

A complex contact manifold (or more properly a holomorphic contact manifold, see the contribution of D. Kotschick in this volume) is a complex manifold of odd complex dimension $2 n+1$ together with an open covering $\left\{\mathcal{O}_{\alpha}\right\}$ by coordinate neighborhoods such that:

1. On each $\mathcal{O}_{\alpha}$ there is a holomorphic 1-form $\theta_{\alpha}$ such that

$$
\theta_{\alpha} \wedge\left(d \theta_{\alpha}\right)^{n} \neq 0 .
$$

2. On $\mathcal{O}_{\alpha} \cap \mathcal{O}_{\beta} \neq \emptyset$ there is a non-vanishing holomorphic function $f_{\alpha \beta}$ such that $\theta_{\alpha}=f_{\alpha \beta} \theta_{\beta}$.

The subspaces $\left\{X \in T_{m} \mathcal{O}_{\alpha}: \theta_{\alpha}(X)=0\right\}$ define a non-integrable holomorphic subbundle $\mathcal{H}$ of complex dimension $2 n$ called the complex contact subbundle or horizontal subbundle. The quotient $L=T M / \mathcal{H}$ is a complex line bundle over $M$. Kobayashi [70] proved that $c_{1}(M)=(n+1) c_{1}(L)$ and hence for a compact complex contact manifold, a complex contact structure is given by a global 1-form if and only if its first Chern class vanishes. It is for this reason that our definition of complex contact structure is analogous to that of a contact structure (in the wider sense). Even for the most canonical example of a complex contact manifold, $\mathbb{C} P^{2 n+1}$, the structure is not given by a global form.

Examples complex contact metric manifolds include the complex Heisenberg group $H_{\mathbb{C}}$ and the odddimensional complex projective space as we have mentioned and which can also be viewed as the twistor space of quaternionic projective space.

The manifold $\mathbb{C}^{n+1} \times \mathbb{C} P^{n}(16)$ as a complex contact manifold was studied by B. Korkmaz [72] and as the complex analogue of the real contact metric structure on the tangent sphere bundle of Euclidean, $E^{n+1} \times S^{n}(4)$.

A complex contact Lie group is a $(2 n+1)$-dimensional complex Lie group $G$ with a left-invariant holomorphic 1-form $\theta$ such that $\theta \wedge(d \theta)^{n} \neq 0$ on the complex manifold $G$. Define $Z$ in the Lie algebra $\mathfrak{g}$ by $d \theta(Z, \cdot)=0$ and $\theta(Z)=1$. Then $\mathfrak{g}=\mathcal{V} \oplus \mathcal{H}$ with $\mathcal{V}=\langle Z\rangle_{\mathbb{C}} ; Z$ is called the Reeb vector field of $\theta$ and one studies the adjoint representation of $\mathcal{V}$.

In [42] B. Foreman studied 3-dimensional complex homogeneous, complex contact manifolds with a global complex contact form and obtained the following classification. 
Theorem 1. If $M$ is a 3-dimensional complex homogeneous, complex contact manifolds with a global complex contact form, then $M$ is of the form $M=G / \Gamma$ where $G$ is a simply connected 3-dimensional complex Lie group and $\Gamma \subset G$ is a discrete subgroup.

If $G$ is unimodular, then $G$ is one of the following:

(a) $S L(2, \mathbb{C})$, if $\operatorname{rk}(\operatorname{ad}(\mathcal{V}))=2$,

(b) The universal cover of the group of rigid motions of the complex Euclidean plane, if $\operatorname{rk}(\operatorname{ad}(\mathcal{V}))=1$,

(c) $H_{\mathbb{C}}$, if $\operatorname{rk}(\operatorname{ad}(\mathcal{V}))=0$.

If $G$ is not unimodular, then $G$ is solvable; $\operatorname{rk}(a d(V))=1$; and $G$ is one of the following complex Lie groups:

(a) The semi-direct product $G_{\alpha}=\mathbb{C} \times \tau_{\alpha} \mathbb{C}^{2}$, for any $\alpha \in \mathbb{C}^{\star} \backslash 1$, where $\tau_{\alpha}$ is the representation of $\mathbb{C}$ in $G L(2, \mathbb{C})$ given by $\tau_{\alpha}(t)=\left(\begin{array}{cc}e^{-t} & 0 \\ 0 & e^{-\alpha t}\end{array}\right)$,

(b) $G=\left\{\left(\begin{array}{ccc}e^{t} & t e^{t} & u \\ 0 & e^{t} & v \\ 0 & 0 & 1\end{array}\right) \mid t, u, v \in \mathbb{C}\right\}$.

In [44] Foreman obtained the following higher dimensional result.

Theorem 2. Let $(G, \theta)$ be $a(2 n+1)$-dimensional complex contact Lie group $G$ such that ad $(Z): \mathfrak{g} \longrightarrow \mathfrak{g}$ is diagonalizable. If $n>1$, then the universal cover group of $G$ is the semi-direct product $\mathbb{C}^{2 n} \times_{\Omega} \mathbb{C}$ where $\Omega$ is the standard symplectic form on $\mathbb{C}^{2 n}$.

Since a holomorphic $p$-form on a compact Kähler manifold is closed, no compact Kähler manifold has a complex contact structure given by a global contact form. Moreover, Y.-G.Ye [116] showed that a compact Kähler manifold with vanishing first Chern class has no complex contact structure. There are however interesting examples of complex contact manifolds with global complex contact forms, called strict complex contact manifolds.

Concerning strict complex contact manifolds, there is a complex Boothby-Wang theorem. First recall that a complex symplectic manifold or holomorphic symplectic manifold is a complex manifold of complex dimension $2 n$ together with a closed holomorphic 2-form $\Omega$ such that $\Omega^{n} \neq 0$. It is important to note that this is not a Kähler manifold and should not be thought of as a manifold that is both complex and symplectic. The key difference here is that the 2-form is holomorphic where as a Kähler form is of bidegree $(1,1)$.

The study of a complex Boothby-Wang theorem was given by B. Foreman [43] who proved the following theorem and its converse.

Theorem 3. Let $M$ be a complex symplectic manifold with a complex symplectic form $\Omega=\Omega_{1}+i \Omega_{2}$ such that both $\Omega_{1}$ and $\Omega_{2}$ determine integral classes. Then the $\left(S^{1} \times S^{1}\right)$-bundle defined by $\left(\left[\Omega_{1}\right],\left[\Omega_{2}\right]\right) \in H^{2}(M, \mathbb{Z}) \oplus$ $H^{2}(M, \mathbb{Z})$ has a complex contact structure given by a holomorphic connection form whose curvature form is $\Omega$.

Theorem 4. Let $P$ be $a(2 n+1)$-dimensional compact complex contact manifold with a global form $\theta=u-i v$ such that the corresponding vertical vector fields $U$ and $V$ are regular. Then $\theta$ generates a free $\left(S^{1} \times S^{1}\right)$-action on $P$ and $p: P \longrightarrow M$ is a principal $\left(S^{1} \times S^{1}\right)$-bundle over a complex symplectic manifold $M$ such that $\theta$ is a connection form for this fibration and the complex symplectic form $\Omega$ on $M$ is given by $p^{\star} \Omega=d \theta$.

Standard examples include ones for which the base complex symplectic manifold is a complex torus of even complex dimension. 
A complex contact manifold $M$ admits a complex almost contact metric structure, i.e. local real 1-forms $u, v=u \circ J,(1,1)$-tensors $G, H=G J$, unit vector fields $U$ and $V=-J U$ and a Hermitian metric $g$ such that

$$
\begin{gathered}
H^{2}=G^{2}=-I+u \otimes U+v \otimes V, \\
g(G X, Y)=-g(X, G Y), \quad g(U, X)=u(X), \\
G J=-J G, \quad G U=0, \quad u(U)=1,
\end{gathered}
$$

and on the overlaps, the above tensors transform as

$$
\begin{array}{cl}
u^{\prime}=a u-b v, & v^{\prime}=b u+a v, \\
G^{\prime}=a G-b H, & H^{\prime}=b G+a H,
\end{array}
$$

for some functions $a, b$ defined on the overlaps with $a^{2}+b^{2}=1$. The local contact form $\theta$ is $u-i v$ to within a nonvanishing complex-valued function multiple.

Moreover, given a complex contact manifold, a complex almost contact metric structure can be chosen such that

$$
\begin{aligned}
& d u(X, Y)=g(X, G Y)+(\sigma \wedge v)(X, Y), \\
& d v(X, Y)=g(X, H Y)-(\sigma \wedge u)(X, Y)
\end{aligned}
$$

for some 1-form $\sigma$. In this case we say that $M$ has a complex contact metric structure.

On a complex contact metric manifold $M$, we can write $T M=\mathcal{H} \oplus \mathcal{V}$, where $\mathcal{V}$ is the vertical subbundle on $M$, locally spanned by $U$ and $V=-J U$, and is usually assumed to be integrable. In this case $\sigma(X)=$ $g\left(\nabla_{X} U, V\right)$. From now on, we will work with a complex contact metric manifold $M$ with structure tensors $(u, v, U, V, G, H, g)$ and complex structure $J$. In the case of a strict complex contact structure, $u$ and $v$ may be taken globally such that $\theta=u-i v$ and $\sigma=0$.

There are two notions of normality in the literature for complex contact manifolds and involve the use of a complex almost contact structure and its several structure tensors. The first is due to Ishihara and Konishi [67], [68]. However their notion seems to be too strong; among its implications is that the underlying Hermitian manifold $(M, g)$ is Kähler. Thus while indeed one of the canonical examples of a complex contact manifold, the odd-dimensional complex projective space, is normal in this sense, the complex Heisenberg group, is not. B. Korkmaz in her thesis (see [73]) generalized the notion of normality and with this notion of normality both odd-dimensional complex projective space and the complex Heisenberg group with their standard complex contact metric structures are normal.

Ishihara and Konishi [67], [68] introduced a notion of normality for complex contact structures. Their notion is the vanishing of the two tensor fields $S$ and $T$ given by

$$
\begin{gathered}
S(X, Y)=[G, G](X, Y) \\
+2 g(X, G Y) U-2 g(X, H Y) V+2(v(Y) H X-v(X) H Y) \\
+\sigma(G Y) H X-\sigma(G X) H Y+\sigma(X) G H Y-\sigma(Y) G H X, \\
T(X, Y)=[H, H](X, Y) \\
-2 g(X, G Y) U+2 g(X, H Y) V+2(u(Y) G X-u(X) G Y) \\
+\sigma(H X) G Y-\sigma(H Y) G X+\sigma(X) G H Y-\sigma(Y) G H X .
\end{gathered}
$$

B. Korkmaz's generalized notion of normality is the following and we adopt her definition here. A complex contact metric structure is normal if

$$
\begin{gathered}
S(X, Y)=T(X, Y)=0, \text { for every } X, Y \in \mathcal{H}, \\
S(U, X)=T(V, X)=0, \text { for every } X .
\end{gathered}
$$


Even though the definition appears to depend on the special nature of $U$ and $V$, it respects the change in overlaps, $\mathcal{O}_{\alpha} \cap \mathcal{O}_{\beta}$, and is a global notion. With this notion of normality both odd-dimensional complex projective space and the complex Heisenberg group with their standard complex contact metric structures are normal.

Turning to curvature, for a unit vector $X \in \mathcal{H}_{m}$ the plane in $T_{m} M$ spanned by $X$ and

$$
Y=a G X+b H X, a, b \in \mathbb{R}, a^{2}+b^{2}=1
$$

is called a GH-plane section and its sectional curvature, $K(X, Y)$, the $G H$-sectional curvature of the plane section. For a given vector $X, K(X, Y)$ is independent of the vector $Y$ in the plane of $G X$ and $H X$ if and only if $K(X, G X)=K(X, H X)$ and $g\left(R_{X G X} H X, X\right)=0$.

Let $M$ be a normal complex contact metric manifold; if the $G H$-sectional curvature is independent of the choice of $G H$-section at each point, it is constant on the manifold and we say that $M$ is a complex contact space form. Odd-dimensional complex projective space with the Fubini-Study metric of constant holomorphic curvature 4 is of constant $\mathrm{GH}$-sectional curvature 1. The complex Heisenberg group has holomorphic curvature 0 for horizontal and vertical holomorphic sections and constant $G H$-sectional curvature -3 . The curvature tensor and the following theorems were obtained by Korkmaz [73].

Theorem 5. Let $M$ be a normal complex contact metric manifold. Then $M$ has constant $G H$-sectional curvature $c$, if and only if for $X$ horizontal, the holomorphic sectional curvature of the plane spanned by X and JX is $c+3$.

Theorem 6. Let $M$ be a normal complex contact metric manifold of constant $G H$-sectional curvature +1 and satisfying $d \sigma(V, U)=2$, then $M$ has constant holomorphic curvature 4. If, in addition, $M$ is complete and simply connected, then $M$ is isometric to $\mathbb{C} P^{2 n+1}$ with the Fubini-Study metric of constant holomorphic curvature 4.

Korkmaz also introduced the idea of an $\mathcal{H}$-homothetic deformation of a complex contact metric structure and proved the following results.

Theorem 7. Complex projective space $\mathbb{C} P^{2 n+1}$ carries a normal complex contact metric structure with constant $G H$-section curvature $\frac{4}{a}-3$ for every $a>0$.

Theorem 8. A normal complex contact metric manifold with metric $\tilde{g}$ of constant $G H$-sectional curvature $\tilde{c}>$ -3 is $\mathcal{H}$-homothetic to a normal complex contact metric manifold with metric $g$ of constant $G H$-section curvature $c=1$. Moreover, if $d \sigma(\tilde{V}, \tilde{U})=\frac{(\tilde{c}+3)^{2}}{8}$, the metric $g$ is Kähler and has constant holomorphic curvature 4.

In [74] Korkmaz continued her study of the curvature of complex contact metric manifolds and of $\mathcal{H}$ homothetic deformations; in particular she developed a theory of complex $(\kappa, \mu)$-spaces. Since that time there has been considerable further work by many authors involving curvature, homogeneity, symmetry, etc. of complex contact manifolds.

\subsection{Some Older Style Topology}

In [87] S. B. Myers proved that a complete Riemannian Manifold for which Ric $\geq \delta>0$ is compact and has finite fundamental group. In [59] Hasegawa and Seino proved that a K-contact manifold for which Ric $\geq \delta>-2$ is compact.

Theorem 9. A K-contact manifold $M^{2 n+1}$ with Ric $\geq \delta>-2$ is compact.

To prove this, let $\theta$ be the infimum of the eigenvalues of the Ricci tensor of $g$. If $\theta>0, M$ is compact, so assume $0 \geq \theta>-2$ and consider the quadratic $2 n t^{2}+2 t-(\theta+2)=0$. Solutions $t_{1}, t_{2}$ satisfy $t_{1}<0<t_{2}<$ $\frac{\theta+2}{2} \leq 1$. Choosing $a>0$ such that $t_{2} \leq a<\frac{\theta+2}{2} \leq 1$ one computes the Ricci tensor for the corresponding $\mathcal{D}$-homothetically deformed metric. This is positive and the result follows from Myers' theorem. 
The classical sphere theorem states that a compact, simply connected Riemannian manifold whose sectional curvature satisfies $\frac{1}{4}<\sec (g) \leq 1$ is homeomorphic to a sphere (Berger [4] for even dimensions and Klingenberg [69] for odd dimensions). Hamilton [57], by means of Ricci flow techniques, extended the result in the 3-dimensional case to pinching by any positive constant and up to diffeomorphism. Ge and Huang [46] studied this question terms of contact geometry and obtained the following results.

Theorem 10. 1) If the sectional curvature of a closed 3-dimensional contact metric manifold satisfies $\frac{1}{4}<$ $\sec (g) \leq 1$, then the universal cover with the lifted contact structure is contactomorphic $S^{3}$ with its standard Sasakian structure.

2) Let $M$ be an open contact metric 3-manifold of nonnegative sectional curvature and positive sectional curvature on $M \backslash K$ where $K$ is a compact subset of $M$. Then the contact structure is tight.

Some topological results were known much earlier. For example, it is known that a complete, simply connected, Sasakian manifold of Riemannian pinching $>0$ and of constant scalar curvature is $\mathcal{D}$-homothethic to the unit sphere, Moskal [85]. Also Tanno showed that a compact Sasakian manifold of sectional curvature $>-3$ is a homology sphere. Proofs of these two results can be found in the lecture notes "Almost Contact Manifolds" by Sasaki [99] along with a number of other noteworthy results.

In the 1960s a great deal of work was done on the topology of compact Sasakian manifolds. The idea was to see how much a compact Sasakian manifold must be like a sphere by studying its Betti numbers. In the case of a compact Kähler manifold, the even-dimensional Betti numbers are different from zero and the odd-dimensional Betti numbers are even. Furthermore the Betti numbers $b_{p}$ of a compact Kähler manifold of positive constant holomorphic curvature are equal to 1 for $p$ even and vanish for $p$ odd, properties which are enjoyed by complex projective space..

The main idea behind the proofs of such results is the Hodge-de Rham theory which we describe briefly here. Let $M$ be a compact orientable manifold without boundary. Recall that for the exterior differential operator on differential forms one has $d^{2}=0$ which leads to the de Rham $p$-dimensional cohomology group of closed $p$-forms modulo exact $p$-forms. We denote this group by $D^{p}\left(M^{n}\right)$. The de Rham theorem asserts that for any $0 \leq p \leq n$, the de Rham cohomology groups are isomorphic to the $p$-th cohomology group, $H^{p}\left(M^{n}, \mathbb{R}\right)$, of the manifold.

On a compact orientable Riemannian manifold without boundary $M^{n}$ one first has an inner product of $p$-forms

$$
(\alpha, \beta)=\int_{M^{n}} \alpha \wedge \star \beta
$$

where * is the Hodge star isomorphism. We also have the co-differential acting on $p$-forms, $\delta=(-1)^{n p+n+1 \star} d^{\star}$ which depends on the metric since ${ }^{\star}$ does and the Laplacian, $\Delta=d \delta+\delta d$. A $p$-form $\alpha$ is said to be harmonic if $\Delta \alpha=0$. Next we have the Hodge deomposition theorem that for a compact orientable Riemannian manifold without boundary and with $\Omega^{p}$ denoting the space of differential $p$-forms

$$
\Omega^{p}=d \Omega^{p-1} \oplus \delta \Omega^{p+1} \oplus H^{p}
$$

where $H^{p}$ is the space of harmonic $p$-forms.

The important upshot of all this is that we have the following isomorphisms

$$
H^{p}\left(M^{n}, \mathbb{R}\right) \cong D^{p}\left(M^{n}\right) \cong H^{p}
$$

and hence the dimension of these groups is the $p$-th Betti number of $M^{n}$.

The Laplacian acting on $p$-forms depends highly on the curvature. Note the curvature terms in the local expression of the Lapacian on a $p$-form $\alpha$.

$$
(\Delta \alpha)_{i_{1} \cdots i_{p}}=-g^{i j} \nabla_{j} \nabla_{i} \alpha_{i_{1} \cdots i_{p}}+\sum_{q=1}^{p} \alpha_{i_{1} \cdots i_{q-1} j i_{q+1} \cdots i_{p}} R_{i_{q}}^{j}+\frac{1}{2} \sum_{r=1}^{p} \sum_{q=1}^{p} \alpha_{i_{1} \cdots i_{q-1} j j i_{q+1} \cdots i_{r-1} i i_{r+1} \cdots i_{p}} R_{i_{q} i_{r}}^{i j}
$$


Tachibana [101] proved that the first Betti number of a compact Sasakian manifold $M^{2 n+1}$ is zero or even. This is proved by first showing that on a compact K-contact manifold, a harmonic 1-form $\omega$ is orthogonal to the contact form $\eta$. Then letting $\tilde{\omega}=\omega \circ \phi$ and computing the Laplacian of $\tilde{\omega}$ one obtains the harmonicity of $\tilde{\omega}$ as well. Thus the number of independent harmonic 1-forms is even. The computation uses the fact that on a Sasakian manifold the Ricci operator commutes with $\phi$. More generally the $p$-th Betti number is even for $p$ odd and $1 \leq p \leq n$ and by duality for $p$ even and $n+1 \leq p \leq 2 n$ (Fujitani [45]; Goldberg and B. [12]).

Considerable attention has been given to the vanishing of the second Betti number under some curvature restrictions as well as being isometric to the unit sphere under stronger conditions. A compact Sasakian manifold of strictly positive curvature has vanishing second Betti number, Moskal [85]. A compact, simplyconnected Sasakian Einstein space of strictly positive curvature is isometric to the unit sphere (Moskal [85]). Pinching theorems have been obtained by Tanno [106] including an analogue of holomorphic pinching.

In [52] Goldberg showed that a compact simply-connected regular Sasakian manifold $M$ of strictly positive curvature is homeomorphic to a sphere. Goldberg had shown earlier [51] that if in addition, $M$ has constant scalar curvature, then $M$ is isometric to a sphere, but not necessarily with a constant curvature metric.

Allowing some negative curvature, Tanno [106] showed that if $M^{2 n+1}$ is a compact K-contact manifold with sectional curvature greater than $\frac{-3}{2 n-1}$, then $b_{1}=0$. Similarly if the Ricci tensor $\rho$ is such that $\rho+2 g$ is positive definite, then $b_{1}=0$. By duality in dimension 3 , one also has $b_{2}=0$.

In dimension 5, Perrone [91] showed that if $M^{5}$ is a compact simply-connected regular Sasakian manifold with $b_{2}=0$ and with scalar curvature $\tau>-4$, then $M^{5}$ is homeomorphic to a sphere. If, in addition, $M^{5}$ has constant scalar curvature, $M$ is isometric to a sphere (but not necessarily with a constant curvature metric).

\section{Curvature Functionals and Ricci Solitons}

The study of the integral of the scalar curvature, $A(g)=\int_{M} \tau d V_{g}$, as a functional on the set $\mathcal{M}_{1}$ of all Riemannian metrics of the same total volume on a compact orientable manifold $M$ is now classical, dating back to Hilbert [61]. A Riemannian metric $g$ is a critical point of $A(g)$ if and only if $g$ is an Einstein metric.

Other functions of the curvature have been taken as integrands as well, most notably $B(g)=\int_{M} \tau^{2} d V_{g}$, $C(g)=\int_{M}|\rho|^{2} d V_{g}$ where $\rho$ is the Ricci tensor, and $D(g)=\int_{M}\left|R_{k j i h}\right|^{2} d V_{g}$; the critical point conditions for these have been computed by Berger [5]. From the critical point conditions it is easy to see that Einstein metrics are critical for $B(g)$ and $C(g)$. However for $C(g)$ there exist non-Einstein critical metrics, Yamaguchi and Chūman [114]. In the case of $B(g)$, Yamaguchi and Chūman showed that a Sasakian critical point is Einstein.

Metrics of constant curvature and Kähler metrics of constant holomorphic curvature are critical for $D(g)$, Muto [86]; also a Sasakian manifold of dimension $2 n+1$ and constant $\phi$-sectional curvature $2(3 n+1)$ is critical for $D(g)$, Yamaguchi and Chūman [114].

Since there are so many Riemannian metrics on a manifold, one can regard, philosophically, the finding of critical metrics as an approach to searching for the best metric for the given manifold. While there seems to be limited success in actually finding the critical metrics, many people have suggested a Ricci flow approach. For example, R. Ye [115] proved that suitably Ricci pinched stable Riemannian metrics on a compact manifold can be deformed to Einstein metrics by means of the Ricci flow.

Here we first consider curvature functionals restricted to spaces of associated metrics rather than the larger class of metrics of the same total volume and then discuss Ricci solitons which several authors have studied in the context of contact metric geometry.

This section will also, at least to some extent, deal with the symplectic case. To set the notation, we write $\Omega(X, Y)=g(X, J Y)$ where $\Omega$ is the symplectic form; the associated metric $g$ and almost complex structure $J$ can be created by polarization as discussed in Section I. We also remark that, as in the contact case, the space $\mathcal{A}$ of associated metrics is infinite dimensional. 
We will often denote by the same letter a tensor field of type $(0,2)$ and its corresponding types $(1,1)$ and $(2,0)$ determined by the metric under consideration, e.g. we may write $\operatorname{tr} T D=T^{i}{ }_{j} D^{j}{ }_{i}=T^{i j} D_{j i}$.

The approach to these critical point problems is to differentiate the functional in question along a path of metrics in the set $\mathcal{M}_{1}$ or for us the set $\mathcal{A}$ of associated metrics. Let $g(t)$ be a path of metrics in $\mathcal{M}_{1}$ or $\mathcal{A}$ and

$$
D_{i j}=\left.\frac{\partial g_{i j}}{\partial t}\right|_{t=0}
$$

its tangent vector at $g=g(0)$. Define two other tensor fields by

$$
\begin{gathered}
D_{j i}{ }^{h}=\frac{1}{2}\left(\nabla_{j} D_{i}{ }^{h}+\nabla_{i} D_{j}{ }^{h}-\nabla^{h} D_{j i}\right) \\
D_{k j i}{ }^{h}=\nabla_{k} D_{j i}{ }^{h}-\nabla_{j} D_{k i}{ }^{h}
\end{gathered}
$$

where $\nabla$ denotes the Levi-Civita connection of $g(0)$ and we note that

$$
D_{j i}{ }^{h}=\left.\frac{\partial \Gamma_{j i}{ }^{h}}{\partial t}\right|_{t=0}, \quad D_{k j i}{ }^{h}=\left.\frac{\partial R_{k j i}{ }^{h}}{\partial t}\right|_{t=0}
$$

where $\Gamma_{j i}{ }^{h}$ and $R_{k j i}{ }^{h}$ denote the Christoffel symbols and curvature tensor of $g(t)$.

We will consider integral functionals defined on the set of metrics associated to a symplectic or contact form. A symmetric tensor field $D$ is tangent to a path in $\mathcal{A}$ at $g$ if and only if

$$
D J+J D=0
$$

in the symplectic case, $J$ being the corresponding almost complex structure, and

$$
D Z_{\eta}=0, \quad D \phi+\phi D=0
$$

in the contact case. For critical point problems on $\mathcal{A}$ the following lemma is important.

Lemma 1. Let $T$ be a second order symmetric tensor field on $M$. Then $\int_{M} T^{i j} D_{i j} d V=0$ for all symmetric tensor fields $D$ satisfying $D J+J D=0$ in the symplectic case and $D Z_{\eta}=0, D \phi+\phi D=0$ in the contact case if and only if $T J=J T$ in the symplectic case and $\phi T-T \phi=\eta \otimes \phi T Z_{\eta}-(\eta \circ T \phi) \otimes Z_{\eta}$ in the contact case (i.e. $\phi$ and $T$ commute when restricted to the contact subbundle).

Now if we consider the functional $A(g)$ restricted to the set $\mathcal{A}$ and seek the critical point condition, since $\mathcal{A}$ is a smaller set of metrics than $\mathcal{M}_{1}$, we expect a weaker critical point condition than that of being Einstein. The critical point condition is that the Ricci operator commutes with the corresponding almost complex structure, still a very natural condition. In particular we have the following result of S. Ianus and the author [13].

Theorem 1. Let $M$ be a compact symplectic manifold and $\mathcal{A}$ the set of metrics associated to the symplectic form. Then $g \in \mathcal{A}$ is a critical point of $A(g)=\int_{M} \tau d V_{g}$ restricted to $\mathcal{A}$ if and only if the Ricci operator $Q$ of $g$ commutes with the almost complex structure corresponding to $g$.

The proof is to compute $\frac{d A}{d t}$ at $t=0$ for a path $g(t)$ in $\mathcal{A}$. Since all associated metrics have the same volume element, this is easier than in the Riemannian case. In particular we have,

$$
\begin{gathered}
\left.\frac{d A}{d t}\right|_{t=0}=\left.\frac{d}{d t} \int_{M} R_{k j i}{ }^{k} g^{j i} d V_{g}\right|_{t=0} \\
=\int_{M} D_{k j i}{ }^{k} g^{j i}-\rho_{j i} D^{j i} d V_{g} \\
=-\int_{M} \rho^{j i} D_{j i} d V_{g},
\end{gathered}
$$


since $D_{k j i}{ }^{k}{ }^{j i}=\left(\nabla_{k} D_{j i}{ }^{k}\right) g^{j i}=\nabla_{k}\left(D_{j i}{ }^{k}{ }^{j i}\right)$ which is a divergence; note that $\operatorname{tr} D=0$ and hence $D_{k i}{ }^{k}=0$. Setting $\left.\frac{d A}{d t}\right|_{t=0}=0$, the result follows from the lemma.

In almost Hermitian geometry we have the ${ }^{\star}$-Ricci tensor and the ${ }^{*}$-scalar curvature defined by

$$
\rho_{i j}^{\star}=R_{i k l t} J^{k l} J_{j}{ }^{t}, \tau^{\star}=\rho_{i}^{\star}{ }^{\star} .
$$

On a Kähler manifold $\rho_{i j}^{\star}=\rho_{i j}$. The most important property of $\tau^{\star}$ on an almost Kähler manifold is

$$
\tau-\tau^{\star}=-\frac{1}{2}|\nabla J|^{2} .
$$

Therefore $\tau-\tau^{\star} \leq 0$ with equality holding if and only if the metric is Kähler. Thus for $M$ compact, Kähler metrics are maxima of the functional

$$
K(g)=\int_{M} \tau-\tau^{\star} d V
$$

on $\mathcal{A}$ and hence it is natural to ask for the critical point condition in general. This was the main question considered in [13]; the critical point condition for $K(g)$ turns out to be the same as for $A(g)$ on $\mathcal{A}$.

Theorem 2. Let $M$ be a compact symplectic manifold and $\mathcal{A}$ the set of metrics associated to the symplectic form. Then $g \in \mathcal{A}$ is a critical point of $K(g)$ if and only if $Q J=J Q$.

It is also natural to ask whether Kähler metrics are the only critical points of $K(g)$; the answer to this is negative and a counterexample was given on the twistor space of a compact Einstein, self-dual 4-manifold with negative scalar curvature by Davidov and Muškarov [35].

At first it may seem surprising that $A(g)$ and $K(g)$ have the same critical point condition but we will see in the course of our discussion that this is natural. The proof of Theorem 2 is then an easy consequence of Theorem 1 and Theorem 3 below though this is not the original proof. The original proof and some work with D. Perrone led the author to consider the "total scalar curvature"

$$
I(g)=\int_{M} \tau+\tau^{\star} d V .
$$

Theorem 3. Let $M^{2 n}$ be a compact symplectic manifold. Then $\int_{M^{2 n}} \tau+\tau^{\star} d V$ is a symplectic invariant and to within a constant is the cup product

$$
\left(c_{1}\left(M^{2 n}\right) \cup[\Omega]^{n-1}\right)\left(\left[M^{2 n}\right]\right)
$$

where $c_{1}\left(M^{2 n}\right)$ is the first Chern class of $M^{2 n}$.

Let us briefly indicate the proof. The generalized Chern form is given by

$$
8 \pi \gamma_{i j}=-4 J^{k}{ }_{j} \rho_{i k}^{*}-J^{k l}\left(\nabla_{j} J^{h}{ }_{k}\right) \nabla_{i} J_{l h} .
$$

Now on an almost Kähler manifold $\left(\nabla_{k} J_{i p}\right) J_{j}^{p}=\left(\nabla_{p} J_{i j}\right) J_{k}{ }^{p}$; this is the condition for an almost Hermitian structure to be quasi-Kähler. Using this, direct computation yields

$$
8 \pi \gamma_{i j} j^{j i}=4 \tau^{\star}-|\nabla J|^{2},
$$

but $\tau-\tau^{\star}=-\frac{1}{2}|\nabla J|^{2}$ and hence $2\left(\tau+\tau^{\star}\right)=8 \pi \gamma_{i j} j^{j i}$. Thus the "total scalar curvature" of an associated metric becomes $I(g)=4 \pi \int_{M^{*}} \gamma_{i j}{ }^{j i} d V$ and integration gives Theorem 3 .

Now writing $\tau-\tau^{\star}$ as $2 \tau-\left(\tau+\tau^{\star}\right)$ we see that $A(g)$ and $K(g)$ have the same critical point condition proving Theorem 2.

In contact geometry the ${ }^{\star}$-Ricci tensor $\rho^{\star}$ and ${ }^{*}$-scalar curvature $\tau^{\star}$ are defined by

$$
\rho_{i j}^{\star}=R_{i k l m} \phi^{k l} \phi_{j}{ }^{m}, \quad \tau^{\star}=\rho_{i}^{*}
$$

and Z. Olszak [90] proved the following theorem. 
Theorem 4. On a contact metric manifold $M^{2 n+1}$

$$
\tau^{\star}-\tau+4 n^{2}=\operatorname{tr} h^{2}+\frac{1}{2}\left(|\nabla \phi|^{2}-4 n\right) \geq 0
$$

with equality if and only if $M^{2 n+1}$ is Sasakian.

The study of $\int_{M} \tau+\tau^{\star} d V$ in symplectic geometry was motivated by the corresponding study in contact geometry. It is interesting to remark that many results in contact and Sasakian geometry were motivated by the corresponding ones in symplectic and Kähler geometry. Here we have an example of a result in contact geometry preceding its symplectic analogue. In contact geometry the functional $I(g)=\int_{M} \tau+\tau^{\star} d V$ is not an invariant and gives a critical point problem whose critical point condition gives the class of K-contact metrics. The proof of this by D. Perrone and the author [15] follows the earlier proof of Theorem 2.

Theorem 5. Let $M$ be a compact contact manifold and $\mathcal{A}$ the set of metrics associated to the contact form. Then $g \in \mathcal{A}$ is a critical point of $I(g)=\int_{M} \tau+\tau^{\star} d V$ if and only if $g$ is K-contact.

There are a few other results concerning the functional $A(g)$ in the contact case, but let's now turn to another functional.

The integral, $L(g)=\int_{M} \operatorname{Ric}\left(Z_{\eta}\right) d V$ was studied in general dimension as the author's first excursion into this area [8] and independently by Chern and Hamilton [29] in the 3-dimensional case. Recall that $\operatorname{Ric}\left(Z_{\eta}\right)=$ $2 n-\operatorname{tr} h^{2}$; thus K-contact metrics, when they occur, are maxima for $L(g)$ on $\mathcal{A}$. Moreover the critical point question for $L(g)$ is the same as that for $\int_{M}|h|^{2} d V$ or $\int_{M}|T|^{2} d V$ where $T(X, Y)=\left(\$_{Z_{\eta}} g\right)(X, Y)=2 g(X, h \phi Y)$. It is the integral $E(g)=\int_{M}|T|^{2} d V$ that was studied by Chern and Hamilton on $\mathcal{A}$ regarded as the set of CRstructures on $M$.

Theorem 6. Let $M$ be a compact regular contact manifold and $\mathcal{A}$ the set of metrics associated to the contact form. Then $g \in \mathcal{A}$ is a critical point of $L(g)=\int_{M} \operatorname{Ric}\left(Z_{\eta}\right) d V$ if and only if $g$ is $K$-contact.

One might conjecture this result without the regularity, however we have the following counterexample: The standard contact metric structure on the tangent sphere bundle of a compact manifold of constant curvature -1 is a critical point of $L$ but is not K-contact. On the other hand Tashiro [110], showed that the standard contact metric structure on the tangent sphere bundle of a Riemannian manifold is K-contact if and only if the base manifold is of constant curvature +1 . Let's note the following result.

Theorem 7. Let $T_{1} M$ be the tangent sphere bundle of a compact Riemannian manifold $(M, G)$ and $\mathcal{A}$ the set of all Riemannian metrics associated to its standard contact structure. Then the standard associated metric is a critical point of the functional $L(g)$ if and only if $(M, G)$ is of constant curvature +1 or -1 .

In [36] S. Deng studied the second variation of the functional $L(g)$ or equivalently, of $E(g)=\int_{M}|T|^{2} d V$, and proved the following.

Theorem 8. Let $g \in \mathcal{A}$ be a critical point of $E(g)$, then $g$ is a minimum.

One often encounters the condition that $Z_{\eta}$ is an eigenvector of the Ricci operator $Q$. One of the more important interpretations of this condition is that of an H-contact manifold as introduced by D. Perrone [93]. First, on a compact $m$-dimensional Riemannian manifold $(M, g)$, a unit vector field $X$ is said to be a harmonic vector field (C. M. Wood [113]) if it is a critical point of the energy functional

$$
\mathcal{E}(X)=\frac{m}{2} \operatorname{vol}(M, g)+\frac{1}{2} \int_{M}\|\nabla X\|^{2} d V
$$

on the space of all unit vector fields. Perrone defines an $H$-contact manifold to be a contact metric manifold for which the Reeb vector field $Z_{\eta}$ is a harmonic vector field, though he just uses the corresponding critical 
point condition; this condition is often referred to as the tension of a harmonic map or harmonic vector field. He therefore does not need compactness in the following theorem.

Theorem 9. A contact metric manifold is an H-contact manifold if and only if its Reeb vector field is an eigenvector of the Ricci operator.

Note that from $\nabla_{X} Z_{\eta}=-\phi X-\phi h X$, if we consider the energy $\mathcal{E}$ as a functional on $\mathcal{A}$ for a fixed contact form, and hence a fixed unit vector field $Z_{\eta}$, then $g \in \mathcal{A}$ is critical for $\mathcal{E}$ if and only if it is critical for the functional $L$. Perrone also proves the following.

Theorem 10. Let $\left(M^{2 n+1}, \eta, g\right)$ be a compact $H$-contact manifold such that $g$ is critical for L. If $\rho+c g$ is positive definite for some constant $c<2-\frac{|\tau|}{\sqrt{2 n}}$, the first Betti number of $M^{2 n+1}$ vanishes.

Furthermore in dimension 3, Perrone showed that a compact H-contact 3-manifold such that $g$ is critical for $L$ is either Sasakian or locally isometric to a non-Sasakian left invariant contact metric structure on $S L(2, \mathbb{R})$ and conversely. In particular, using the classification of Geiges mentioned in Section I, Perrone showed that a compact $\mathrm{H}$-contact 3-manifold such that $g$ is critical for $L$ is diffeomorphic to a left invariant quotient of $S U(2)$, the Heisenberg group or $\widetilde{S L}(2, \mathbb{R})$ by a discrete group.

Ever since the work of Hamilton and especially Perelman's proof of the Poincare conjecture, there has been considerable interest in the Ricci flow and its applications. A number of people have raised the question of how interesting this might be in the context of contact metric geometry. There have been a number of results on Ricci solitons. We will give a brief introduction to this topic here.

One starts with a Riemannian manifold $\left(M, g_{0}\right)$ and seeks to evolve the metric to a path or 1-parameter family $g(t), g(0)=g_{0}$, satisfying

$$
\frac{\partial g(t)}{\partial t}=-2 \rho,
$$

that is the metric evolves at a rate proportional to its Ricci tensor $\rho$. A path $g(t)$ is a self-similar solution of the Ricci flow evolution equation if there exist scalars $\sigma(t)$ and diffeomorphisms $\psi_{t}$ of $M$ such that $g(t)=\sigma(t) \psi_{t}^{\star} g_{0}$.

Now consider a Riemannian manifold $\left(M, g_{0}\right)$ such that

$$
-2 \rho_{0}=\$_{X} g_{0}+2 \lambda g_{0}
$$

holds for some constant $\lambda$ and some complete vector field $X$ on $M$. In this case $g_{0}$ is said to be a Ricci soliton; some authors regard the manifold with such a metric as the Ricci solition and we will often take this point of view here. By rescaling one may assume that $\lambda \in\{-1,0,1\}$; these three cases correspond to shrinking, steady and expanding Ricci solitons.

In the case that the vector field $X$ is the gradient of a potential function $-f$, one has

$$
\nabla \nabla f=\rho+\lambda g
$$

and $g_{0}$ is said to be a gradient Ricci soliton. A gradient Ricci soliton is said to be rigid if it is isometric to a quotient of $N \times E^{k}$ where $N$ is an Einstein manifold and $f(x)=\frac{\lambda}{2}|x|^{2}$ on the Euclidean factor. Euclidean space $E^{n}$ gives a trivial steady Ricci soliton by taking $X=0$. Also $E^{n}$ can be regarded as an expanding gradient Ricci soliton, called the Gaussian soliton, by taking $\lambda=1$ and choosing the potential function. $f(x)=\frac{1}{2}|x|^{2}$.

The key proposition is the following; a proof can be found in the book of B. Chow and D. Knopf ([34] p. 23).

Proposition 1. If $(M, g(t))$ is a self-similar solution of the Ricci flow equation, then there exists a vector field $X$ on $M$ such that $\left(M, g_{0}, X\right)$ is a Ricci solition. Conversely, given a Ricci soliton $\left(M, g_{0}, X\right)$, there exists 1-parameter families of scalars $\sigma(t)$ and diffeomorphisms $\psi_{t} \operatorname{such}(M, g(t))$ is a solution of the Ricci flow equation of the form $g(t)=\sigma(t) \psi_{t}^{\star} g_{0}$. 
In our discussion of Lie groups we considered in some detail the Lie group of rigid motions of Minkowski plane and in the section on curvature we spent a fair amount of time on $(\kappa, \mu)$-manifolds. Setting $\lambda=1$ in that discussion, we have the associated metric

$$
g=\frac{1}{4}\left(2 e^{2 y} d x^{2}+d y^{2}+2 e^{-2 y} d z^{2}\right)
$$

for the contact form

$$
\eta=\frac{1}{2}\left(e^{y} d x+e^{-y} d z\right) .
$$

Direct computation of the curvature shows that $E(1,1)$ with this structure is a $(0,4)$-manifold. Then for the vector field $X=-8 x \frac{\partial}{\partial x}-8 z \frac{\partial}{\partial z}$ we have

$$
\$_{X} g+2 \rho+16 g=0 .
$$

Therefore $(E(1,1), g, X)$ is a Ricci soliton. With this example in mind, let us state the following theorem which is a combination of results by Ghosh and Sharma [50] and of Cho, Hashinaga, Kubo, Taketomi, Tamaru [33].

Theorem 11. Let $\left(M^{2 n+1}, \eta, g\right)$ be a connected, simply connected, complete non-Sasakian $(\kappa, \mu)$-manifold. Then $\left(M^{2 n+1}, \eta, g\right)$ is a Ricci soliton if and only if it is the 3-dimensional Gaussian soliton, the gradient shrinking Ricci soliton $E^{n+1} \times S^{n}(4)$ or a non-gradient expanding Ricci soliton on a $(0,4)$-manifold. The latter case can be realized as a homogeneous real hypersurface of the Grassmannian $G_{2}^{\star}\left(\mathbb{R}^{n+3}\right)$.

J. T. Cho [32] also introduces a contact Ricci soliton as a solution to a contact Ricci flow problem. The resulting equation is

$$
\$_{Z_{\eta}} g+2 \rho-2 \lambda g=0 \text {; }
$$

Cho then proves that a contact Ricci soliton is shrinking and is Einstein K-contact. Now Boyer and Galicki [25] proved that a compact Einstein K-contact manifold is Sasakian; therefore we have as a corollary that a compact contact Ricci solition is Sasakian Einstein.

We should also return to the Sasakian case and discuss some recent work on the Sasakian Einstein metrics using the idea of a transverse Ricci flow. This idea was introduced by Smoczyk, Wang and Zhang [100]. Their notation is quite different than what we have been using; we will continue to use $\rho$ for the Ricci tensor. First of all a contact metric structure is said to be $\eta$-Einstein if its Ricci tensor $\rho$ is of the form $\rho=\alpha g+\beta \eta \otimes \eta$ where $\alpha$ and $\beta$ are constants. Define a transverse metric $g^{T}$ by

$$
g^{T}=g-\eta \otimes \eta
$$

Let $\pi: \mathcal{D} \oplus \mathbb{R} Z_{\eta} \longrightarrow \mathcal{D}$ be the natural projection and define a transverse Levi-Civita connection $\nabla^{T}$ by

$$
\nabla_{X}^{T} Y= \begin{cases}\pi\left(\nabla_{X} Y\right) & \text { if } X \in \Gamma(\mathcal{D}) \\ \pi([X, Y]) & \text { if } X \in \Gamma\left(\mathbb{R} Z_{\eta}\right)\end{cases}
$$

Let $R_{X Y}^{T} Z$ denote the corresponding curvature tensor and $\rho^{T}$ the transverse Ricci tensor, i.e.

$$
\rho^{T}(X, Y)=g\left(R_{X e_{i}}^{T} e_{i}, Y\right)
$$

for $X, Y \in T M$ and where $\left\{e_{i}\right\}$ is an orthonormal basis of $\mathcal{D}$.

In the context of transverse Riemannian foliations, Lovrić, Min-Oo and Ruh [80] introduced a transverse Ricci flow by

$$
\frac{\partial g(t)^{T}}{\partial t}=-\rho^{T}
$$

which could be considered in the Sasakian setting. However, it is not easy to check directly that the flow preserves the Sasakian character. Therefore Smoczyk, Wang and Zhang introduce a Sasakian Ricci flow by

$$
\frac{\partial g(t)^{T}}{\partial t}=-\left(\rho^{T}-\kappa g^{T}\right) .
$$

This first leads to the following theorem. 
Theorem 12. Let $M^{2 n+1}$ be a compact Sasakian manifold with structure tensors, $\left(\phi, Z_{\eta}, \eta, g\right)$. Then there is a family of Sasakian structures $\left(\phi(t), Z_{\eta}(t), \eta(t), g(t)\right), t \in[0, T)$ for some constant $T$ with $Z_{\eta}(t)=Z_{\eta}$ constituting a Sasakian Ricci flow and initial condition $(\phi(0), \eta(0), g(0))=(\phi, \eta, g)$.

The main result of Smoczyk, Wang and Zhang, which involves some quite difficult analysis, is the following.

Theorem 13. For the Sasakian Ricci flow on a compact manifold we have long-time existence of the solution. Moreover when the basic first Chern class is negative or null, the Sasakian Ricci flow converges to an $\eta$-Einstein metric.

A Sasakian manifold $M^{2 n+1}$ is a gradient Sasakian Ricci soliton if there exists a basic function $f$ (i.e. $Z_{\eta} f=0$ ) satisfying

$$
\rho^{T}+\operatorname{Hess}^{T} f=(2 n+2) g^{T} .
$$

H. Tadano [104] gave a number of results characterizing Sasakian Einstein manifolds; for example we have the following.

Theorem 14. Let $M$ be a (2n+1)-dimensional compact gradient Sasakian Ricci soliton. Then $M$ is a Sasakian Einstein manifold if and only if

$$
\rho_{\text {max }}^{T}-2 n(2 n+2) \leq\left(1+\frac{1}{n}\right) \mathcal{F}
$$

where $\mathcal{F}=\frac{1}{\text { volM }} \int_{M}\left\|\nabla^{T} f\right\|^{2} d V$ is the Sasakian Futaki invariant.

\section{Associated Metrics for more than one Contact Form}

It is sometimes asked if a Riemannian metric $g$ can be an associated metric for more than one contact structure and we have the following result of Tachibana and $\mathrm{Yu}$ [103].

Theorem 1. If $\left(M^{2 n+1}, g\right)$ is a complete Riemannian manifold which admits two Sasakian structures $(\eta, g)$ and $\left(\eta^{\prime}, g\right)$ with $\eta \neq \pm \eta^{\prime}$, then either $\left(M^{2 n+1}, g\right)$ is covered by the standard Sasakian structure on $S^{2 n+1}$ or $n=2 k+$ $1,\left(M^{4 k+3}, g\right)$ is 3-Sasakian and $\left(\eta, \eta^{\prime}\right)$ belong to an $S^{2}$-family of Sasakian structures with $g$ as their common associated metric. Moreover if the angle function of the two Reeb vector fields, $g\left(Z_{\eta}, Z_{\eta^{\prime}}\right)$ is non-constant then only the first case can occur.

An other result in this style is result of Apostolov, Draghici and Moroianu [1], namely

Theorem 2. Let $\left(M^{2 n+1}, \eta, g\right)$ be a Sasakian Einstein manifold. Then any contact metric structure, $\left(\eta^{\prime}, g\right)$, with Reeb vector field $Z_{\eta^{\prime}}$ and the same metric is Sasakian. Moreover if $Z_{\eta^{\prime}} \neq Z_{\eta}$, then either $\left(M^{2 n+1}, g\right)$ is covered by a round sphere or it admits a 3-Sasakian structure.

More recently Draghici and Rukimbira proved the following three theorems [40].

Theorem 3. Let $\left(M^{3}, g\right)$ be a connected Riemannian manifold of constant scalar curvature. Assume that $\left(M^{3}, g\right)$ admits a Sasakian structure $(\eta, g)$ and another contact metric structure $\left(\eta^{\prime}, g\right)$ with $\eta \neq \pm \eta^{\prime}$. Then either $\left(\eta^{\prime}, g\right)$ is also Sasakian and $\left(M^{3}, g\right)$ is the standard unit sphere or $\left(\eta^{\prime}, g\right)$ is not Sasakian with $\eta$ and $\eta^{\prime}$ inducing opposite orientations and $\left(M^{3}, g\right)$ is locally isometric to the Lie group $\widetilde{S L}(2, \mathbb{R})$.

The structure on $\widetilde{S L}(2, \mathbb{R})$ used in this theorem is slightly different from that we discussed earlier. 
Theorem 4. Let $\left(M^{2 n+1}, \eta, g\right)$ be a compact Sasakian manifold which admits another K-contact structure $\left(\eta^{\prime}, g\right)$ with $\left[Z_{\eta}, Z_{\eta^{\prime}}\right]=0$. Set $f=g\left(Z_{\eta}, Z_{\eta^{\prime}}\right)$ and assume that $f^{-1}(1)$ or $f^{-1}(-1)$ has codimension 2. Then $\left(\eta^{\prime}, g\right)$ is also Sasakian and is covered by a round sphere.

In dimension 5, Draghici and Rukimbira give the following generalization of the theorem of Tachibana and Yu.

Theorem 5. Suppose $\left(M^{5}, \eta, g\right)$ is a complete Sasakian manifold admitting another $K$-contact structure $\left(\eta^{\prime}, g\right)$. Then $\left(\eta^{\prime}, g\right)$ is also Sasakian and if $Z_{\eta} \neq \pm Z_{\eta^{\prime}}$, then $\left(M^{5}, g\right)$ is covered by a round sphere.

Instead of just treating two contact forms, we should also discuss contact circles, especially taut contact metric circles as introduced recently by D. Perrone [94]. First, however, a pair $\left\{\eta_{1}, \eta_{2}\right\}$ of contact forms on a 3-dimensional manifold is called a contact circle if for every $a=\left(a_{1}, a_{2}\right) \in S^{1} \subset \mathbb{R}^{2}, \eta_{a}=a_{1} \eta_{1}+a_{2} \eta_{2}$ is also a contact form, Geiges and Gonzalo [48]. If the volume forms $\eta_{a} \wedge\left(d \eta_{a}\right)^{n}$ are equal for all $a \in S^{1}$, the pair $\left\{\eta_{1}, \eta_{2}\right\}$ is called a taut contact circle. A taut contact circle is called a Cartan structure if both $\eta_{1} \wedge d \eta_{2}$ and $\eta_{2} \wedge d \eta_{1}$ vanish.

Perrone then defines a taut contact metric circle to be a triple $\left(\eta_{1}, \eta_{2}, g\right)$ where $\left(\eta_{1}, \eta_{2}\right)$ is taut and $g$ is an associated metric for both $\eta_{1}$ and $\eta_{2}$. Perrone also introduces the notion of a bi-contact metric structure as a triple $\left(\eta_{1}, \eta_{2}, g\right)$ where $\left(\eta_{1}, \eta_{2}\right)$ is a pair of contact forms and $g$ is an associated metric for both of them and such that the corresponding almost contact structures satisfy

$$
\phi_{1} \phi_{2}+\varepsilon \eta_{1} \otimes Z_{\eta_{2}}=-\left(\phi_{2} \phi_{1}+\varepsilon \eta_{2} \otimes Z_{\eta_{1}}\right)
$$

where $\varepsilon= \pm 1$ is defined by $\phi_{2} Z_{\eta_{1}}=\varepsilon \phi_{1} Z_{\eta_{2}}$. We now have the following results from Perrone [94].

Theorem 6. Let $\left(\eta_{1}, \eta_{2}\right)$ be a pair of contact forms and $g$ a Riemannian metric on a 3-manifold. Then $\left(\eta_{1}, \eta_{2}, g\right)$ is a taut contact metric circle if and only if it is a bi-contact metric structure with $\varepsilon=-1$.

Theorem 7. Let $\left(\eta_{1}, \eta_{2}\right)$ be a pair of contact forms on a 3-manifold. Then $\left(\eta_{1}, \eta_{2}\right)$ is a Cartan structure if and only if there exists a Riemannian metric $g$ such that $\left(\eta_{1}, \eta_{2}, g\right)$ is a taut contact metric circle.

In his paper Perrone also classifies simply connected 3-manifolds admititng bi-H-contact metric structures (i.e. both structures are H-contact, see section 5); these are the Lie groups $S U(2), \widetilde{S L}(2, \mathbb{R}), \widetilde{E}(2), E(1,1)$. As in Theorem 3 above the orientation of the forms $\eta_{1}$ and $\eta_{2}$ plays a role. In particular, if $\eta_{1}$ and $\eta_{2}$ induce the same orientation, the Lie groups $S U(2), \widetilde{S L}(2, \mathbb{R}), \widetilde{E}(2)$ arise; if the opposite orientation is induced only $\widetilde{S L}(2, \mathbb{R})$ and $E(1,1)$ occur. It is interesting to note that in the case of opposite orientations, the vector cross product $Z_{\eta_{1}} \times Z_{\eta_{2}}$, which defines the common kernel of $\eta_{1}$ and $\eta_{2}$, induces a conformally Anosov flow on any compact quotient of $\widetilde{S L}(2, \mathbb{R})$ and $E(1,1)$.

\section{References}

[1] V. Apostolov, T. Draghici and A. Moroianu, The odd-dimensional Goldberg conjecture, Math. Nachr. 279 (2006), $948-952$.

[2] V. I. Arnol'd, First steps in symplectic topology, Russian Math. Surveys 41 (1986), 1-21.

[3] K. Bang and D. E. Blair, The Schouten tensor and conformally flat manifolds, Topics in Differential Geometry, Ed. Acad. Române, Bucharest (2008), 1-28.

[4] M. Berger, Sur quelques variétés riemanniennes suffisament pincées, Bull. Soc. Math. France 88 (1960), 57-71.

[5] M. Berger, Quelques formules de variation pour a structure riemannienne, Ann. Sc. École Norm. Sup. 3 (1970), $285-294$.

[6] D. E. Blair, On the non-existence of flat contact metric structures, Tôhoku Math. J. 28 (1976), 373-379.

[7] D. E. Blair, Two remarks on contact metric structures, Tôhoku Math. J. 29 (1977), 319-324.

[8] D. E. Blair, Critical associated metrics on contact manifolds, J. Austral. Math. Soc. Series A 37 (1984), 82-88.

[9] D. E. Blair, The "total scalar curvature" as a symplectic invariant and related results, Proc. 3rd Congress of Geometry Thessaloniki (1991), 79-83.

[10] D. E. Blair, Riemannian Geometry of Contact and Symplectic Manifolds, Second Edition, Birkhäuser, Boston, 2010. 
[11] D. E. Blair and A. Carriazo, The contact Whitney sphere, Note di Matematica 20 (2000), 125-133.

[12] D. E. Blair and S. I. Goldberg, Topology of almost contact manifolds, J. Differential Geom. 1 (1967), 347-354.

[13] D. E. Blair and S. Ianus, Critical associated metrics on symplectic manifolds, Contemp. Math. 51 (1986), 23-29.

[14] D. E. Blair, Th. Koufogiorgos and B. J. Papantoniou, Contact metric manifolds satisfying a nullity condition, Israel J. Math. 91 (1995), 189-214.

[15] D. E. Blair and D. Perrone, A variational characterization of contact metric manifolds with vanishing torsion, Canad. Math. Bull. 35 (1992), 455-462.

[16] E. Boeckx, A class of locally $\phi$-symmetric contact metric spaces, Arch. Math. 72 (1999), 466-472.

[17] E. Boeckx, A full classification of contact metric $(k, \mu)$-spaces, Illinois J. Math. 44 (2000), 212-219.

[18] E. Boeckx, Contact-homogeneous locally $\varphi$-symmetric manifolds, Glasgow Math. J. 48 (2006), 93-109.

[19] E. Boeckx, P. Bueken and L. Vanhecke, $\phi$-symmetric contact metric spaces, Glasgow Math. J. 41 (1999), 409-416.

[20] E. Boeckx and J. T. Cho, Locally symmetric contact metric manifolds, Monatsh. Math. 148 (2006), 269-281.

[21] W. M. Boothby, Homogeneous complex contact manifolds, Proc. Symp. Pure Math. III, Amer. Math. Soc. (1961), 144-154.

[22] W. M. Boothby, A note on homogeneous complex contact manifolds, Proc. Amer. Math. Soc. 13 (1962), 276-280.

[23] W. M. Boothby and H. C. Wang, On contact manifolds, Ann. of Math. 68 (1958), 721-734.

[24] V. Borrelli, B.-Y. Chen and J.-M. Morvan, Une caractérisation géométrique de la sphère de Whitney, C. R. Acad. Sci. Paris 321 (1995), 1485-1490.

[25] C. P. Boyer and K. Galicki, Einstein manifolds and contact geometry, Proc. Amer. Math. Soc. 129 (2001), 2419-2430.

[26] G. Calvaruso, D. Perrone and L. Vanhecke, Homogenneity on three-dimensional contact metric manifolds, Israel J. Math. 114 (1999), 301-321.

[27] B. Cappelleti Montano and L. Di Terlizzi, Contact metric $(\kappa, \mu)$-spaces as bi-Legendrian manifolds, Bull. Austral. Math. Soc. 77 (2008), 373-386.

[28] S. S. Chern, Pseudo-groupes continus infinis, colloques Internationaux du C. N. R. S., Strasbourg (1953), 19-136.

[29] S. S. Chern and R. S. Hamilton, On Riemannian metrics adapted to three-dimensional contact manifolds, Lecture Notes in Mathematics Vol. 1111 (1985), Springer, Berlin, 279-308.

[30] D. Chinea, Invariant submanifolds of a quasi-K-Sasakian manifold, Riv. Mat. Univ. Parma 11 (1985), 25-29.

[31] J. T. Cho, A class of contact Riemannian manifolds whose associated CR-structures are integrable, Publ. Math. Debrecen 63 (2003), 193-211.

[32] J. T. Cho, Notes on contact Ricci solitons, Proc. Edinb. Math. Soc. 54 (2011), 47-53.

[33] J. T. Cho, T. Hashinaga, A. Kubo, Y. Taketomi and H. Tamaru, Realizations of some contact metric manifolds as Ricci soliton real hypersurfaces, J. Geom. Phys. 123 (2018), 221-234.

[34] B. Chow and D. Knopf, The Ricci Flow: An Introduction, Mathematical Surveys and Monographs, Vol. 110, Amer. Math. Soc., Providence (2004).

[35] J. Davidov and O. Muškarov, Twistor spaces with Hermitian Ricci tensor, Proc. Amer. Math. Soc. 109 (1990), 1115-1120.

[36] S. Deng, The second variation of the Dirichlet energy on contact manifolds, Kōdai Math. J. 14 (1991), 470-476.

[37] F. Dillen and L. Vrancken, C-totally real submanifolds of $S^{7}(1)$ with non-negative sectional curvature, Math. J. Okayama Univ. 31 (1989), 227-242.

[38] F. Dillen and L. Vrancken, C-totally real submanifolds of Sasakian space forms, J. Math. Pures Appl. 69 (1990), 85-93.

[39] P. Dombrowski, On the geometry of the tangent bundle, J. reine und angew. Math. 210 (1962), 73-88.

[40] T. Draghici and P. Rukimbira, Sasakian metrics with an additional contact structure, African Diaspora Journal of Mathematics 14 (2012), 1-15

[41] H. Endo, Invariant submanifolds in contact metric manifolds, Tensor (N.S.) 43 (1985), 86-89.

[42] B. Foreman, Three-dimensional complex homogeneous complex contact manifolds, Balkan J. Geom. and Appl. 4 (1999), 53-67.

[43] B. Foreman, Boothby-Wang fibrations on complex contact manifolds, Differential Geom. Appl. 13 (2000). 179-196.

[44] B. Foreman, Complex contact Lie groups and generalized complex Heisenberg groups, Differential Geom. Appl. 24 (2006), 443-446.

[45] T. Fujitani, Complex-valued differential forms on normal contact Riemannian manifolds, Tôhoku Math. J.18 (1966), $349-361$.

[46] J. Ge and Y. Huang, 1/4-pinched contact sphere theorem, Asian J. math. 20 (2016), 893-901.

[47] H. Geiges, Normal contact structures on 3-manifolds, Tôhoku Math. J. 49 (1997), 415-422.

[48] H. Geiges and J. Gonzalo, Contact geometry and complex surfaces, Invent. math. 121 (1995), 147-209.

[49] H. Geiges and J. Gonzalo, An application of convex intergration to contact geometry, Trans. Amer. Math. Soc. 348 (1996), 2139-2149.

[50] A. Ghosh and R. Sharma, A classification of Ricci solitons as $(k, \mu)$-contact metrics, Springer Proc. Math. Stat., Vol. 106, (2014), 349-358.

[51] S. I. Goldberg, Rigidity of positively curved contact manifolds, J. London Math. Soc. 42 (1967), 256-263.

[52] S. I. Goldberg, Non-negatively curved contact manifolds, Proc. Amer. Math. Soc. 96 (1986), 651-656

[53] J. Gonzalo, Branched covers and contact structures, Proc. Amer. Math. Soc. 101 (1987), 347-352.

[54] J. Gray, Some global properties of contact structures, Ann. of Math. 69 (1959), 421-450.

[55] S. Greenfield, Cauchy-Riemann equations in several variable, Ann. Scuola Norm. Sup. Pisa 22 (1968), 275-314. 
[56] M. Gromov, Pseudo holomorphic curves in symplectic manifolds, Invent. Math. 82 (1985), 307-347.

[57] R. S. Hamilton, Three-manifolds with positive Ricci curvature, J. Differential Geom. 17 (1982), 255-306.

[58] M. Harada, On Sasakian submanifolds, Tôhoku Math. J. 25 (1973),103-109.

[59] I. Hasegawa, and M. Seino, Some remarks on Sasakian geometry, J. Hokkaido Univ. Education 32 (1981), 1-7.

[60] Y. Hatakeyama, On the existence of Riemann metrics associated with a 2-form of rank 2r, Tôhoku Math. J. 14 (1962), $162-166$.

[61] D. Hilbert, Die grundlagen der physik, Nachr. Ges. Wiss. Göttingen (1915), 395-407.

[62] C. J. Hsu, On some structures which are similar to the quaterion structure, Tôhoku Math. J. 12 (1960), 403-428.

[63] M. Hutchings and C. H. Taubes, Proof of the Arnold chord conjecture in three dimensions I, Math. Res. Lett. 18 (2011), 295313.

[64] M. Hutchings and C. H. Taubes, Proof of the Arnold chord conjecture in three dimensions II, Geom. Topol. 17 (2013), 26012688.

[65] C. Huygens, Traité de la Lumiere, Vander Aa, Leiden (1690).

[66] S. Ianus, Sulle varietà di Cauchy-Rieman, Rend. dell'Accademia di Scienze Fisiche e Matematiche Napoli, XXXIX (1972), 191195.

[67] S. Ishihara and M. Konishi, Real contact 3-structure and complex contact structure, Southeast Asian Bulletin of Math. 3 (1979), 151-161.

[68] S. Ishihara and M. Konishi, Complex almost contact manifolds, Kōdai Math. J. 3 (1980), 385-396.

[69] W. Klingenberg, Über riemannsche Mannigfaltigkeiten mit positiver Krümmung, Comment. Math. Helv. 34 (1961), $47-54$.

[70] S. Kobayashi, Remarks on complex contact manifolds, Proc. Amer. Math. Soc. 10 (1959), 164-167.

[71] S. Kobayashi and K. Nomizu, Foundations of Differential Geometry, I, Wiley-Interscience, New York (1963).

[72] B. Korkmaz, A curvature property of complex contact metric structures, Kyungpook Math. J. 38 (1998), 473-488.

[73] B. Korkmaz, Normality of complex contact manifolds, Rocky Mountain J. Math. 30 (2000), 1343-1380.

[74] B. Korkmaz, A nullity condition for complex contact manifolds, J. Geom. 77 (2003),108-128.

[75] Th. Koufogiorgos, Contact Riemannian manifolds with constant $\phi$-sectional curvature, Tokyo J. Math. 20 (1997), 13-22.

[76] Th. Koufogiorgos and C. Tsichlias, On the existence of a new class of contact metric manifolds, Canad. Math. Bull.43 (2000), 440-447.

[77] V. Krouglov, A note on the conjecture of Blair in contact Riemannian Geometry, Tôhoku Math. J. 64 (2012), 561-567.

[78] S. Lie, Theorie der Transformationgruppen, Vol. 2, Leipzig, Teubner (1890).

[79] A. Lotta, Non-existence of homogeneous contact metric manifolds of non-positive curvature, Tôhoku Math. J. 62 (2010), 575-578.

[80] M. Lovrić, M. Min-Oo and E. A. Ruh, Deforming transverse Riemannian metrics of foliations, Asian J. Math. 4 (2000), $303-$ 314.

[81] J. Martinet, Formes de contact sur les variétiés de dimension 3, Lecture Notes in Mathematics Vol. 209 Springer, Berlin (1971), 142-163.

[82] J. Milnor, Curvature of left invariant metrics on Lie groups, Advances in Math. 21 (1976), 293-329.

[83] G. Mitric, C. R.-structures on the unit sphere bundle in the tangent bundle of a Riemannian manifold, Semin. Mec. Timis. Fac. Mat. 32 (1991).

[84] K. Mohnke, Holomorphic disks and the Chord Conjecture, Annals of Mathematics 154 (2001), 219-222.

[85] E. Moskal, Contact Manifolds of Positive Curvature, Thesis, University of Illinois, 1966.

[86] Y. Muto, Critical Riemannian metrics, Tensor (N.S.) 29 (1975), 125-133.

[87] S. B. Myers, Riemannian manifolds with positive mean curvature, Duke Math. J. 8 (1941), 401-404.

[88] K. Ogiue, On almost contact manifolds admitting axiom of planes or axiom of free mobility, Kōdai. Math. Sem. Rep. 16 (1964),115-128.

[89] M. Okumura, Some remarks on space with a certain contact structure, Tôhoku Math. J. 14 (1962), 135-145.

[90] Z. Olszak, On contact metric manifolds, Tôhoku Math. J. 31 (1979), 247-253.

[91] D. Perrone, 5-dimensional contact manifolds with second Betti number $b_{2}=$ 0, Tôhoku Math. J. 41 (1989), 163-170.

[92] D. Perrone, Homogeneous contact Riemannian three-manifolds, Ill. J. Math. 42 (1998), 243-256.

[93] D. Perrone, Contact metric manifolds whose characteristic vector field is a harmonic vector field, Differential Geom. Appl. 20 (2004), 367-378.

[94] D. Perrone, Taut contact circles and bi-contact metric structures on three-manifolds, Ann. Global Anal. Geom. 52 (2017), 213-235.

[95] D. Perrone and L. Vanhecke, Five-dimensional homogeneous contact manifolds and related problems, Tôhoku Math. J. 43 (1991), 243-248.

[96] G. Reeb, Sur certaines propriétés topologiques des trajectoires des systèmes dynamiques, Mémoires de l'Acad. Roy. de Belgique, Sci. Ser. 2, 27 (1952), 1-62.

[97] A. Ros, Positively curved Kaehler submanifolds, Proc. Amer. Math. Soc. 93 (1985), 329-331.

[98] P. Rukimbira, A characterization of flat contact metric geometry, Houston Math. J. 24 (1998), 409-414.

[99] S. Sasaki, Almost Contact Manifolds, Lecture Notes, Mathematical Institute, Tôhoku University, Part I (1965), Part II (1967), Part III (1968).

[100] K. Smoczyk, G. Wang and Y. Zhang, The Sasaki-Ricci flow, Internat. J. Math. 21 (2010), 951-969. 
[101] S. Tachibana, On harmonic tensors in compact Sasakian spaces, Tôhoku Math. J. 17 (1965), 271-284.

[102] S. Tachibana and M. Okumura, On the almost complex structure of tangent bundles of Riemannian spaces, Tôhoku Math. J. 14 (1962), 156-161.

[103] S. Tachibana and W. N. Yu, On a Riemannian space admitting more than one Sasakian structure, Tôhoku Math. J. 29 (1970), 536-540.

[104] H. Tadano, Gap theorems for compact gradient Sasaki-Ricci solitons, Internat. J. Math. 26 (2015), 17pp.

[105] T. Takahashi, Sasakian $\phi$-symmetric spaces, Tôhoku Math. J. 29 (1977), 91-113.

[106] S. Tanno, The topology of contact Riemannian manifolds, Illinois J. Math. 12 (1968), 700-717.

[107] S. Tanno, Geodesic flows on $C_{L}$-manifolds and Einstein metrics on $S^{3} \times S^{2}$, Minimal Submanifolds and Geodesics, Kaigai, Toyko (1978), 283-292.

[108] S. Tanno, Variational problems on contact Riemannian manifolds, Trans. Amer. Math. Soc. 314 (1989), 349-379.

[109] S. Tanno, The standard CR-structure on the unit tangent bundle, Tôhoku Math. J. 44 (1992), 535-543.

[110] Y. Tashiro, On contact structures of tangent sphere bundles, Tôhoku Math. J. 21 (1969), 117-143.

[111] D. Van Lindt, P. Verheyen and L. Verstraelen, Minimal submanifolds in Sasakian space forms, J. Geom. 27 (1986), 180-187.

[112] J. A. Wolf, Complex homogeneous contact manifolds and quaternionic symmetric spaces, J. Math. and Mech. 14 (1965), 1033-1047.

[113] C. M. Wood, On the energy of a unit vector field, Geometria Dedicata 64 (1997), 319-330.

[114] S. Yamaguchi and G. Chūman, Critical Riemannian metrics on Sasakian manifolds, Kōdai, Math. J 6 (1983), 1-13.

[115] R. Ye, Ricci flow, Einstein metrics and space forms, Trans. Amer. Math. Soc. 338 (1993), 871-896.

[116] Y.-G. Ye, A note on complex projective threefolds admitting holomorphic contact structures, Invent. Math. 115 (1994), 311 314.

[117] A. Zeghib, Subsystems of Anosov systems, Amer. J. Math. 117 (1995), 1431-1448. 